\title{
DNA priming and gp120 boosting induces HIV-specific antibodies in a randomized clinical trial
}

\author{
Nadine G. Rouphael, ${ }^{1}$ Cecilia Morgan, ${ }^{2}$ Shuying S. Li, ${ }^{2}$ Ryan Jensen, ${ }^{2}$ Brittany Sanchez, ${ }^{2}$ Shelly Karuna, ${ }^{2}$ Edith Swann, ${ }^{3}$ \\ Magdalena E. Sobieszczyk, ${ }^{4}$ Ian Frank, ${ }^{5}$ Gregory J. Wilson, ${ }^{6}$ Hong-Van Tieu, ${ }^{7}$ Janine Maenza, ${ }^{2,8}$ Aliza Norwood, ${ }^{9}$ \\ James Kobie, ${ }^{10}$ Faruk Sinangil, ${ }^{11}$ Ciuseppe Pantaleo, ${ }^{12}$ Song Ding, ${ }^{13}$ M. Juliana McElrath, ${ }^{2}$ Stephen C. De Rosa, ${ }^{2}$ \\ David C. Montefiori, ${ }^{14}$ Guido Ferrari, ${ }^{14}$ Georgia D. Tomaras,,${ }^{14}$ Michael C. Keefer, ${ }^{10}$ and the HVTN 105 Protocol Team \\ and the NIAID HIV Vaccine Trials Network ${ }^{15}$
}

\begin{abstract}
'Hope Clinic of the Emory Vaccine Center, Division of Infectious Diseases, Emory University, Atlanta, Georgia, USA. ${ }^{2}$ Vaccine and Infectious Disease Division, Fred Hutchinson Cancer Research Center, Seattle, Washington, USA. ${ }^{3}$ Division of AIDS, NIH, Bethesda, Maryland, USA. ${ }^{4}$ Columbia University Medical Center, New York, New York, USA. ${ }^{5}$ University of Pennsylvania, Philadelphia, Pennsylvania, USA. ${ }^{6}$ Vanderbilt University Medical Center, Nashville, Tennessee, USA. ${ }^{~} N e w$ York Blood Center, New York, New York, USA. ${ }^{8}$ University of Washington, Seattle, Washington, USA. ${ }^{~}$ UCSF, San Francisco, California, USA. ${ }^{10}$ Department of Medicine, University of Rochester School of Medicine \& Dentistry, Rochester, New York, USA. "Clobal Solutions for Infectious Diseases, South San Francisco, California, USA. ${ }^{2}$ Division of Immunology and Allergy, Lausanne University Hospital (CHUV), Lausanne, Switzerland. ${ }^{13 E}$ uroVacc Foundation, Lausanne, Switzerland. ${ }^{14}$ Department of Surgery, Duke University Medical Center, Durham, North Carolina, USA. ${ }^{15 T}$ The HVTN 105 Protocol Team and the NIAID HIV Vaccine Trials Network is detailed in the Supplemental Acknowledgments.
\end{abstract}

BACKCROUND. RV144 is the only preventive HIV vaccine regimen demonstrating efficacy in humans. Attempting to build upon RV144 immune responses, we conducted a phase 1, multicenter, randomized, double-blind trial to assess the safety and immunogenicity of regimens substituting the DNA-HIV-PT123 (DNA) vaccine for ALVAC-HIV in different sequences or combinations with AIDSVAX B/E (protein).

METHODS. One hundred and four HIV-uninfected participants were randomized to 4 treatment groups (T1, T2, T3, and T4) and received intramuscular injections at $0,1,3$, and 6 months $(M)$ : T1 received protein at $M 0$ and $M 1$ and DNA at M3 and M6; T2 received DNA at $\mathrm{MO}$ and $\mathrm{M} 1$ and protein at $\mathrm{M3}$ and $\mathrm{M6}$; $\mathrm{T3}$ received DNA at $\mathrm{M0}, \mathrm{M1}, \mathrm{M3}$, and $\mathrm{M6}$ with protein coadministered at M3 and M6; and T4 received protein and DNA coadministered at each vaccination visit.

RESULTS. All regimens were well tolerated. Antibodies binding to gp120 and V1V2 scaffold were observed in $95 \%-100 \%$ of participants in T3 and T4, two weeks after final vaccination at high magnitude. While IgC3 responses were highest in T3, a lower IgA/IgG ratio was observed in T4. Binding antibodies persisted at 12 months in 35\%-100\% of participants. Antibody-dependent cell-mediated cytotoxicity and tier 1 neutralizing-antibody responses had higher response rates for T3 and T4, respectively. CD4 ${ }^{+} \mathrm{T}$ cell responses were detectable in all treatment groups (32\%-64\%) without appreciable CD8 ${ }^{+} \mathrm{T}$ cell responses.

CONCLUSION. The DNA/protein combination regimens induced high-magnitude and long-lasting HIV V1V2-binding antibody responses, and early coadministration of the 2 vaccines led to a more rapid induction of these potentially protective responses.

TRIAL REGISTRATION. ClinicalTrials.gov NCT02207920.

FUNDING. National Institute of Allergy and Infectious Diseases (NIAID) grants UM1 AI068614, UM1 AI068635, UM1 AI068618, UM1 AI069511, UM1 AI069470, UM1 AI069534, P30 Al450008, UM1 AI069439, UM1 Al069481, and UM1 AI069496; the National Center for Advancing Translational Sciences, NIH (grant UL1TR001873); and the Bill \& Melinda Gates Foundation (grant OPP52845).

Related Commentary: p. 4572

Conflict of interest: The authors have declared that no conflict of interest exists. Copyright: (c) 2019 Rouphael et al. This is an open access article published under the terms of the Creative Commons Attribution 4.0 International License.

Submitted: March 12, 2019; Accepted: July 24, 2019; Published: September 30, 2019 Reference information: / Clin Invest. 2019;129(11):4769-4785.

https://doi.org/10.1172/JCl128699.

\section{Introduction}

Almost 40 million people are living with HIV worldwide. Every year, there are 1.8 million new infections and 1.2 million deaths from HIV-related causes (1), making the need for a safe and effective HIV vaccine a public health priority. To date, RV144 is the only vaccine efficacy trial that demonstrated protection against HIV acquisition (2). RV144 was a randomized placebo-controlled trial evaluating a regimen consisting of a replication-defective canarypox vector (ALVAC vCP1521) with HIV gag, protease, and env inserts administered at $0,1,3$, and 6 months, in combination 
Table 1. Vaccination schema of HVTN 105

\begin{tabular}{|c|c|c|c|c|c|c|}
\hline \multirow[b]{2}{*}{ Group } & \multirow[b]{2}{*}{ N } & \multirow[b]{2}{*}{ Deltoid } & \multicolumn{4}{|c|}{ Injection schedule in months (days) } \\
\hline & & & $0(0)$ & $1(28)$ & $3(84)$ & $6(168)$ \\
\hline \multirow[t]{2}{*}{$\mathrm{T} 1$} & 26 & Left & Placebo & Placebo & DNA & DNA \\
\hline & & Right & AIDSVAX B/E & AIDSVAX B/E & Placebo & Placebo \\
\hline \multirow[t]{2}{*}{$\mathrm{T} 2$} & 26 & Left & DNA & DNA & Placebo & Placebo \\
\hline & & Right & Placebo & Placebo & AIDSVAX B/E & AIDSVAX B/E \\
\hline \multirow[t]{2}{*}{ T3 } & 26 & Left & DNA & DNA & DNA & DNA \\
\hline & & Right & Placebo & Placebo & AIDSVAX B/E & AIDSVAX B/E \\
\hline \multirow[t]{2}{*}{ T4 } & 26 & Left & DNA & DNA & DNA & DNA \\
\hline & & Right & AIDSVAX B/E & AIDSVAX B/E & AIDSVAX B/E & AIDSVAX B/E \\
\hline Total & 104 & & & & & \\
\hline
\end{tabular}

with a recombinant gp120 protein (AIDSVAX B/E) administered at 3 and 6 months among 16,402 adults at varying risk of HIV-1 acquisition in Thailand. Prior efficacy trials using AIDSVAX B/E (VAX003, ref. 3) or AIDSVAX B/B alone (VAX004, ref. 4) failed to protect against HIV acquisition, emphasizing the potential importance of a prime-boost regimen.

Vaccine efficacy in RV144, albeit moderate, appeared to be predominantly mediated by antibody responses, based on the results of a case-control study that examined immunogenicity in peripheral blood at the time of peak responses, 2 weeks after the final vaccination at month 6 (5). While vaccine efficacy was $31 \%$ at 36 months, the predetermined time point of primary analysis, early vaccine efficacy at 1 year was $60 \%$, reflecting the kinetics of HIV-specific humoral responses in the peripheral blood (6). IgG antibodies directed against the variable loop region (V1V2) of gp120, including those of the IgG3 subclass, were associated with a lower risk of HIV-1 acquisition $(5,7)$. Specifically, the magnitude of anti-V1V2 IgG responses appeared to be important, as participants with titers in the highest tertile were found to have a vaccine efficacy of $60 \%$ compared with those in the lowest tertile or with negative responses $(5,6)$. In addition, HIV-specific antibody-dependent cell-mediated cytotoxicity (ADCC) in the presence of low HIV-1 envelope-specific (Env-specific) IgA correlated with decreased HIV-1 risk (5). Plasma HIV-1 Env-specific IgA antibodies in vaccine recipients were found to be directly correlated with infection risk in RV144 and were postulated to interfere with protective Fc-mediated antibody functions such as $\operatorname{ADCC}(5,8)$. Thus, while these potential immunologic correlates of HIV infection risk require confirmation in ongoing efficacy trials (i.e., the HVTN 702/Uhambo study currently underway in South Africa), these immunologic markers can be helpful in early-phase studies to assess alternative vaccine strategies to optimize protective responses.

The HIV Vaccine Trials Network (HVTN) 105 trial (ClinicalTrials.gov NCT02207920) was designed to investigate vaccine priming alternatives to the RV144 strategy with a DNA-HIV candidate vaccine replacing the ALVAC vector and additional modifications of the prime-boost sequence. HVTN 105 evaluated the safety and immunogenicity of AIDSVAX B/E (consisting of a 1:1 mixture of gp120 strains $\mathrm{MN}$ and A244 in alum) also used in RV1144, and the DNA plasmid vaccine (DNA-HIV-PT123) contain- ing a clade C (strain ZM96) gp140 envelope that was substituted for the ALVAC vCP1521 but contained the same HIV genes as ALVAC used in RV144. One HVTN 105 group (T3) matched the RV144 prime-boost regimen (DNA-HIV-PT123 at 0, 1, 3, and 6 months with AIDSVAX B/E at 3 and 6 months), while coadministration of both candidate vaccines at all 4 vaccination time points was examined in another group (T4). Another group (T2) matched the RV144 regimen but omitted the third and fourth DNA-HIV injections at 3 and 6 months, and the reverse of that sequence, protein vaccine given as a prime followed by DNA vaccine boosts, was examined in the remaining group (T1).

The DNA component of this experimental vaccine regimen offers several potential advantages over the ALVAC vector in RV144, in that DNA vaccines are thermostable, are relatively straightforward to manufacture, and provide more flexibility for vaccine design through formulation of multiple plasmids containing different HIV components and/or adjuvants in a single injection. DNA HIV vaccines also have a favorable safety profile in large studies (6) in combination with other vector vaccines such as modified vaccinia Ankara (MVA) (9) and adenovirus serotype 5 (10). To date, however, only 2 small HIV vaccine clinical trials have been conducted using combination regimens of DNA and recombinant protein, and these trials showed induction of significant and persistent binding antibodies and $\mathrm{T}$ cell responses $(11,12)$. Furthermore, to the best of our knowledge no human data exist on priming with a protein and boosting with a DNA vaccine and there are limited human data on the coadministration of these products from the initial vaccination, although studies in nonhuman primates show encouraging results (13-17).

The primary goal of the HVTN 105 trial was to utilize the immunologic profile of potential correlates of protection identified in the RV144 efficacy trial to determine (a) whether administration of a DNA-HIV vaccine instead of ALVAC-HIV in the same regimen can induce comparable or superior immune responses, and (b) how the early coadministration of DNA and protein affects the kinetics and character of immune responses over the course of the trial. In addition, HVTN 105 examined how 4 doses versus 2 doses of DNA-HIV priming affects immunogenicity, and whether protein immunization can serve as an effective prime for subsequent boosting with DNA-HIV.

\section{Results}

\section{Study participants, demographics, and vaccine safety}

One hundred and four participants at low risk for HIV acquisition were enrolled at US sites between July 16, 2014 and December 10, 2014, and randomly assigned into 1 of 4 study treatment groups (Table 1). Participant characteristics are described in Supplemental Table 1; supplemental material available online with this article; https://doi.org/10.1172/JCI128699DS1. Fiftythree percent of participants were male, $31 \%$ were non-white, and $10 \%$ were of Latino ethnicity. The median age of participants was 27 years (range 18-50 years). Demographics among 


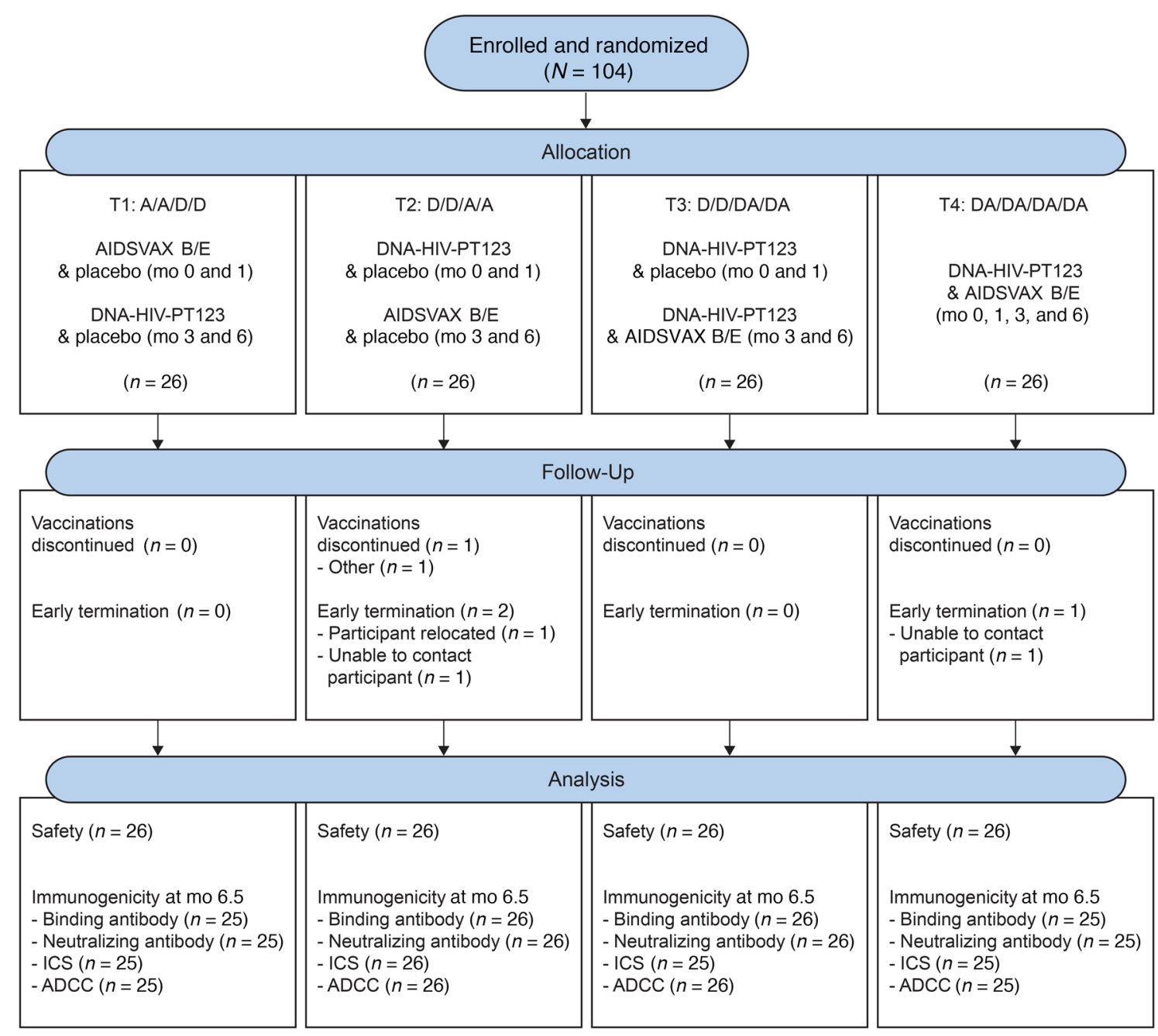

Figure 1. HVTN 105 CONSORT statement flow diagram.

the 4 groups were comparable. Ninety-eight percent of participants (102 of 104) received all 4 vaccinations and the overall retention rate in the trial was high (101 of 104, 97\%); 1 participant (in T2) relocated and 2 participants (1 each in T2 and T4) were unable to be contacted during the follow-up period after receiving all 4 vaccinations (Figure 1 ).

Both DNA-HIV and AIDSVAX B/E vaccines were well tolerated. There were no serious adverse events (SAEs) reported in the study. Eighty-five percent $(n=88)$ of study participants experienced one or more adverse events (AEs), but only 9 (8.7\%) of these participants had 15 AEs that were judged by the investigators to be related to the study agents (decrease in absolute neutrophil count $[n=3]$, elevation in alanine aminotransferase level $[n=3]$, decrease in hemoglobin level $[n=1]$, tenderness/ enlargement of draining axillary lymph node $[n=3]$, pruritus at injection site $[n=2]$, nodule at injection site $[n=1]$, shoulder pain $[n=1]$, and fatigue $[n=3])$. Local (Supplemental Figure 1A) and systemic (Supplemental Figure 1B) reactions, when present, were self-limited, with the majority being of mild to moderate grade. One participant in T4 experienced severe (grade 3) erythema of greater than $9 \mathrm{~cm}$ in diameter at the right deltoid (AIDSVAX B/E) injection site after the second and third vaccinations, and another participant in $\mathrm{T} 2$ reported grade 3 fatigue/malaise after the fourth vaccination. The most frequent local reactions were pain and/or tenderness in $85.6 \%$ (mild in $71.2 \%$, moderate in $14.4 \%$ ) of individuals, and the most frequent systemic reactions were malaise/ fatigue in $40.4 \%$ (mild in $27.9 \%$, moderate in $11.5 \%$, severe $1 \%$ ) of individuals. There were no statistically significant differences across the 4 groups for local or systemic reactions except more mild/moderate headache $(P=0.03)$ in $\mathrm{T} 1$, and no vaccinations were discontinued due to AEs.

\section{Vaccine-induced humoral immunogenicity}

Frequency and magnitude of IgG, IgG subclass, and IgA Env-specific binding antibodies. The total Env-specific binding-antibody responses were evaluated against vaccine strain gp120 antigens (ZM96.C, MN.B, and A244.AE) and consensus envelope antigens (Con S gp140 CFI [group M consensus]) central to all circulating strains, with good sensitivity for HIV vaccine immunogenicity assessment $(7,18,19)$. We also evaluated IgG binding-antibody responses to different conformational V1V2 antigens that correlated with decreased risk of HIV acquisition in RV144, including those binding to C.1086 V1V2, the env sequence strain selected for further clinical trials in sub-Saharan Africa. 


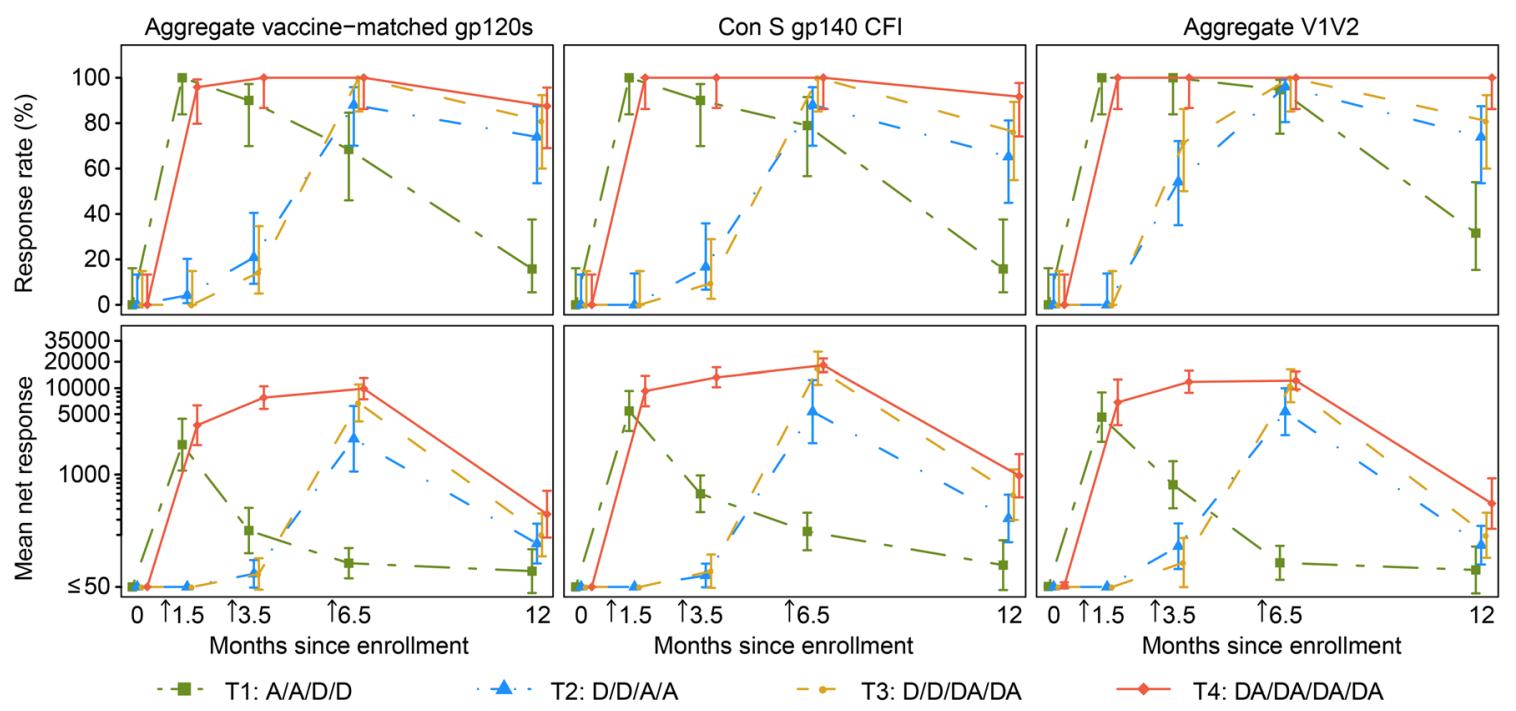

Figure 2. IgG binding-antibody responses in HVTN 105 participants over time, as measured by binding-antibody multiplex assay (BAMA) against aggregate vaccine-matched gp120 antigens, 1 gp140 antigen, and aggregate V1V2 antigens. Shown are the positive-response rates and 95\% Cls estimated using the score test method (top panels) and the geometric mean response magnitudes among all participants and $95 \% \mathrm{Cl}$ based on an assumption of $\log (\mathrm{IgG})$ following a normal distribution (bottom panels) by time point and treatment group $(n=25,26,26,25$ in T1-T4, respectively). The lines connect the response rates/geometric mean magnitudes between time points. Vaccine-matched gp120 antigens: A244.AE, MN.B, and ZM96.C. gp140 antigen: Con S gp140 CFI. V1V2 antigens: 1086.C V1V2, CaseA2_gp70_V1V2.B, CaseA2_V1/V2/169K.B, and A244.AE V1V2. Arrows indicate the second, third, and fourth vaccinations. D, DNA; A, AIDSVAX B/E.

Within each treatment group, trends of binding-IgG response rates and geometric mean response magnitudes were similar over time across HIV antigens (vaccine-matched vs. consensus HIV envelopes, V1V2 antigens). In all treatment groups, response rates of $81 \%-100 \%$ were observed 2 weeks after the second vaccination with AIDSVAX B/E, which occurred at month 1.5 in T1 and $\mathrm{T} 4$ following early administration of protein, as opposed to month 6.5 in T2 and T3 (Figure 2). However, the response rates in $\mathrm{T} 1$ were not sustained at later time points with boosting by DNA alone. Comparing the other treatment groups at month 6.5 ( 2 weeks after the fourth vaccination), there were no significant differences between $\mathrm{T} 3$ and $\mathrm{T} 4$ in IgG response rates to any antigen, but significant differences were observed when $\mathrm{T} 3$ and $\mathrm{T} 4$ were compared to $\mathrm{T} 2$ for vaccine-matched antigens. $\mathrm{T} 4$ response rates were higher than T2 for ZM96.C (100\% vs. $80 \%$, $P=0.05)$ and A244.AE (100\% vs. $80 \%, P=0.05)$ (Supplemental Figure 2). Also, the IgG response rate was significantly higher in T3 than T2 for ZM96.C (100\% vs. 80\%, $P=0.05$ ). Importantly, at this time point, binding-IgG responses to the HIV V1V2 antigens identified in RV144 as potential inverse correlates of risk (A244.AE V1V2 and 1086.C V1V2) were observed in $96 \%$ or more vaccinees in groups $\mathrm{T} 2, \mathrm{~T} 3$, and $\mathrm{T} 4$, with high median response magnitudes (26,881-29,212 for A244.AE V1V2 and 11,723-23,634 for 1086.C V1V2) (Figure 3).

Durable binding-IgG responses, as measured at month 12 (6 months after the fourth vaccination), were demonstrated in over $65 \%$ of participants against the aggregated vaccine-matched and consensus envelope antigens only if they received a boosting regimen that included AIDSVAX B/E (groups T2, T3, and T4) (Figure 2). Although the magnitude of IgG responses against HIV V1V2 significantly decreased by month 12 (6 months after the fourth vaccination) in all groups, they were still detectable in the majority of T3 and T4 participants for A244.AE V1V2 (81\% T3 and 100\% $\mathrm{T} 4)$ and 1086.C V1V2 (67\% T3 and 71\% T4).

Assessment of IgG3 and IgG4 subclass binding to HIV Env and V1V2 was also conducted longitudinally for all treatment groups. At month 6.5 (2 weeks after the fourth vaccination), IgG3 binding to A244.AE V1V2 was observed in the majority of participants in $\mathrm{T} 2$, T3, and $\mathrm{T} 4$ (62\%, 92\%, and 76\%, respectively), with similar findings for the 1086.C V1V2 antigen in these 3 groups (50\%, 64\%, and $44 \%$, respectively) (Figure 4A). Of note, peak IgG3 binding responses were observed 2 weeks after the second vaccination with AIDSVAX B/E, with no significant differences in response rates or magnitude between T3 and T4. Similarly, T1 participants had peak IgG3 responses similar in frequency and magnitude to those of $\mathrm{T} 4$ at month 1.5 , but these responses rapidly waned to become negligible by month 6.5 ( 2 weeks after the second boost with DNAHIV alone). Durability of IgG3 binding responses at month 12 (6 months after the fourth vaccination) was poor, with extremely low or absent responses across all antigens in all treatment groups (Figure 4A and Supplemental Figure 3A). HIV-specific IgG4 responses in groups $\mathrm{T} 1-\mathrm{T} 3$ were low after each vaccination (Figure 4B). In contrast to groups T1-T3, in T4, HIV-specific IgG4 responses were significantly boosted after the third and fourth vaccination. Specifically, the rates of IgG4 response to the A244.AE V1V2 and 1086.C V1V2 antigens at month 6.5 were $84 \%$ and $72 \%$, respectively, among participants in T4, as compared with a range of $15 \%-19 \%$ and $4 \%-15 \%$, respectively, for these antigens in groups $\mathrm{T} 1, \mathrm{~T} 2$, and T3. Also of note, unlike IgG3 responses, IgG4 responses among T4 participants persisted at month 12 (6 months after the fourth vaccination), without a significant decline in response rate or magnitude (Figure 4B and Supplemental Figure 3B).

Serum IgA binding responses to 2 HIV envelope proteins, consensus A gp140 (a direct correlate of risk identified in RV144; refs. 

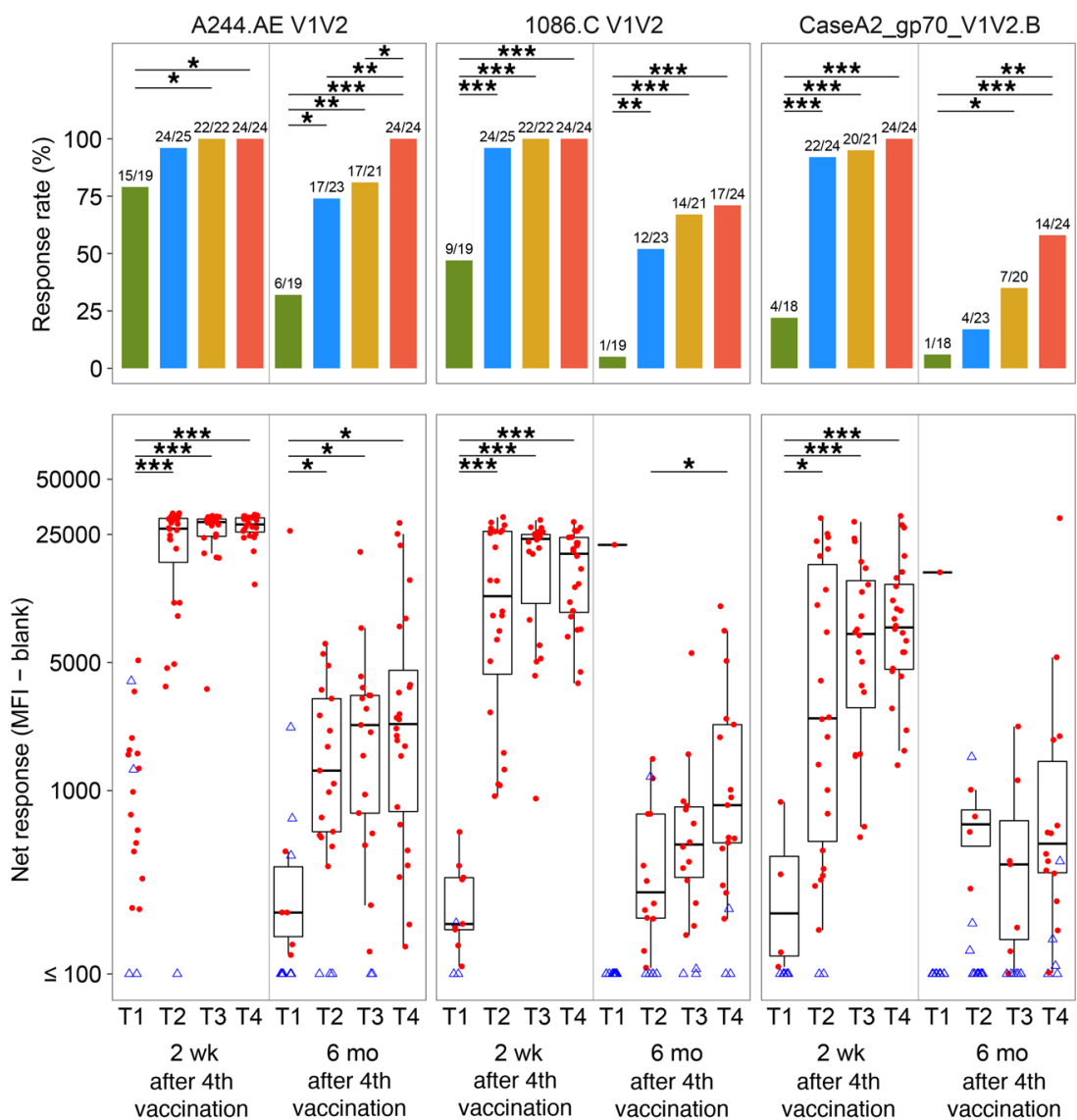

2 wk after 4 th vaccination
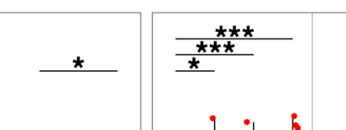

T1: A/A/D/D

T2: D/D/A/A

T3: D/D/DA/DA

T4: DA/DA/DA/DA

Figure 3. IgC binding-antibody responses 2 weeks and 6 months after the fourth vaccination in HVTN 105, as measured by binding-antibody multiplex assay (BAMA) against $\mathbf{3}$ V1V2 antigens. Shown are the positive-response rates (top panels) and the distribution of the response magnitudes (positive responders in red circles and nonresponders in blue triangles) and the box-and-whisker plots among the positive responders (the midline of the box-and-whisker plot denotes the median and the ends of the box-and-whisker plot denote the 25th and 75th percentiles) (bottom panels) by time point and treatment group ( $n=25,26,26,25$ in T1-T4, respectively). V1V2 antigens: A244.AE V1V2, 1086.C V1V2, and CaseA2_gp70_V1V2.B. Bars on the top of plots indicate the significant differences between treatment groups within the same visit $\left({ }^{*} P \leq 0.05 ;{ }^{* *} P\right.$ $\leq 0.01$; ${ }^{* *} P \leq 0.001$ ) without multiple-comparisons adjustment. The comparisons between treatment groups were performed using Fisher's exact test for response rates and Wilcoxon's rank-sum test for magnitudes. Fractions above bars on top panels indicate numbers of positive responses over total numbers of responses (negative and positive) by time point and treatment group. D, DNA; A, AIDSVAX B/E.
5, 8) and A244.AE (an AIDSVAX B/E-matched gp120), were examined longitudinally for all treatment groups (Figure 5). Similarly to IgG, IgA responses for each group peaked 2 weeks after administration of the second protein vaccination (at month 1.5 in $\mathrm{T} 1$ and $\mathrm{T} 4$, and at month 6.5 in $\mathrm{T} 2$ and $\mathrm{T} 3$ ). However, IgA responses were positive in less than $50 \%$ of participants in each group. At month 6.5 ( 2 weeks after the fourth vaccination), the IgA response rate was significantly higher in T3 than T4 for consensus A gp140 and A244.AE (Figure 5A), indicating that serum IgA responses were not boosted by the third and fourth doses of AIDSVAX B/E in T4. The IgA/IgG ratio at this time point was low for both groups, but statistically higher for $\mathrm{T} 3$ compared with $\mathrm{T} 4$ for the A244.AE antigen (Figure 5B). All groups had negligible IgA responses at month 12 (6 months after the fourth vaccination) (Figure 5A).

ADCC. Assessment of ADCC, as measured by the GranToxiLux (GTL) assay, was performed at the month 6.5 and month 12 time points ( 2 weeks and 6 months after the fourth vaccination, respectively) for all treatment groups. ADCC-GTL responses were observed for the 3 vaccine-matched gp120 antigens in T2, T3, and T4 at month 6.5 (Figure 6). Responses to the subtype B antigen, MN.B, in these groups were present in over $90 \%$ of participants, and responses persisted at month 12 in about half of the participants. ADCC-GTL responses to the ZM96.C-coated (subtype C) and A244.AE-coated (subtype A/E) targets were detected in a similar proportion of $\mathrm{T} 2, \mathrm{~T} 3$, and $\mathrm{T} 4$ participants (ranging between $38 \%$ and $56 \%$ ) at the month 6.5 time point; however, negligible responses were observed by month 12 . Finally, ADCC-
GTL responses among T1 group participants were detected only against the subtype B antigen, MN.B, but were significantly less frequent compared with the other 3 groups for this antigen by month 12. The magnitude of ADCC responses measured as area under the curve (AUC) recapitulated the response rate. The highest magnitudes were observed against the MN.B-coated targets in all 4 groups at the month 6.5 and 12 time points, and the AUCs were significantly higher in T3 compared with T4 two weeks, but not 6 months, after the fourth immunization.

Neutralizing-antibody responses. Neutralizing-antibody (nAb) response assays to tier $1 \mathrm{HIV}$ strains were assessed at the month 6.5 and month 12 time points (2 weeks and 6 months after the fourth vaccination, respectively) for all treatment groups. At month 6.5, response rates and magnitudes varied among the 5 tier 1 strains, but tended to be highest for those more closely matched to the vaccine antigens MN.3.B (subtype B), MW965.26.C (subtype C), and TH023.6.AE (subtype A/E) (Figure 7A). Participants in $\mathrm{T} 1$ had significantly lower $\mathrm{nAb}$ response rates and magnitudes than participants in the other groups. Participants in T4 had the strongest responses of the 4 treatment groups for MW965.26.C and TH023.6.AE. Of note, peak rates of $\mathrm{nAb}$ responses to strains MW965.26.C and TH023.6.AE were significantly higher in T4 compared with T3 $(P=0.05$ and 0.0003 , respectively) and T2 $(P=0.002$ and $<0.0001$, respectively). Also, the nAb titers for these 2 strains were significantly higher in $\mathrm{T} 4$ compared with $\mathrm{T} 3$ or $\mathrm{T} 2$ at this time point for the MW965.26.C (T4 vs. T3: $P=0.002$; T4 vs. T2: $P=$ 0.008) and for the TH023.6.C strain (T4 vs. T3: $P<0.0001 ; \mathrm{T} 4$ vs. 
A
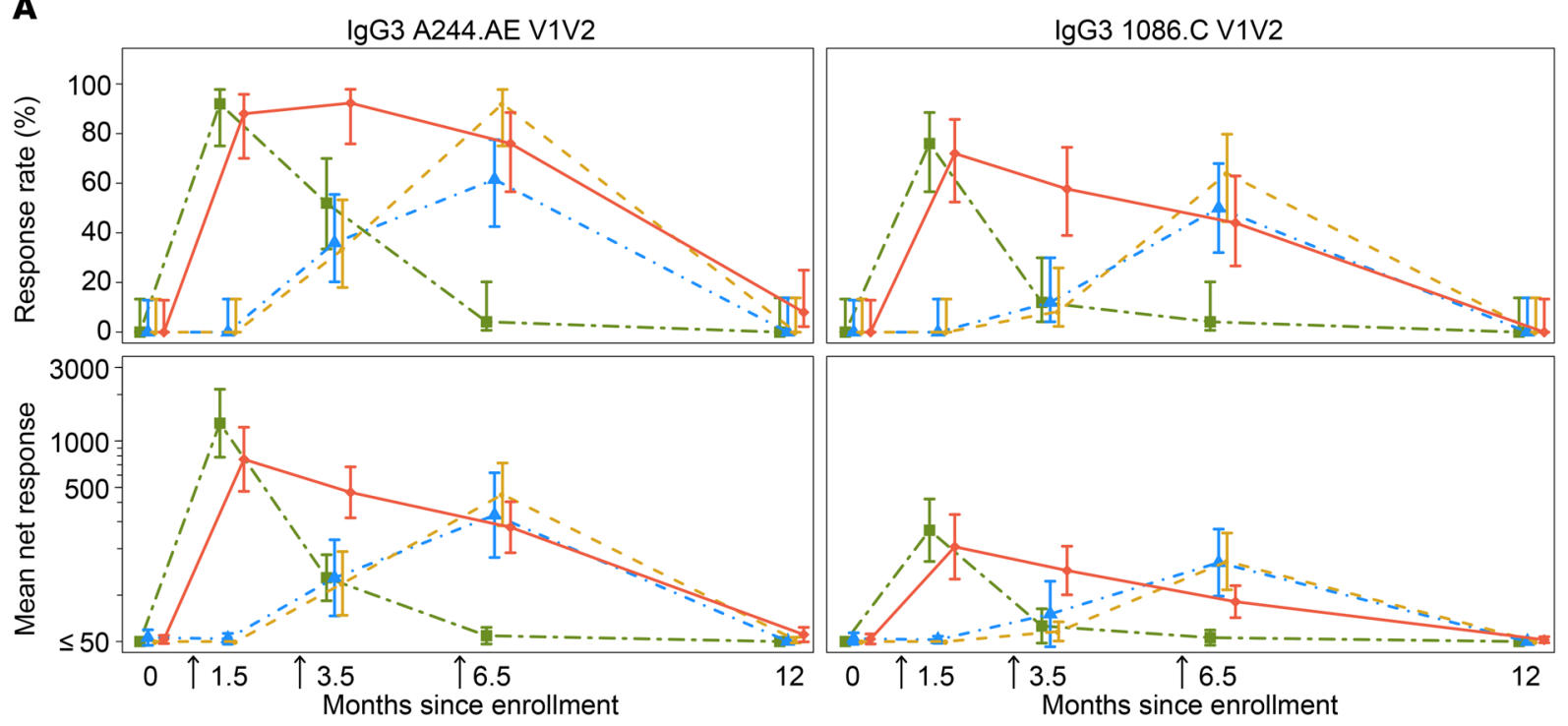

B
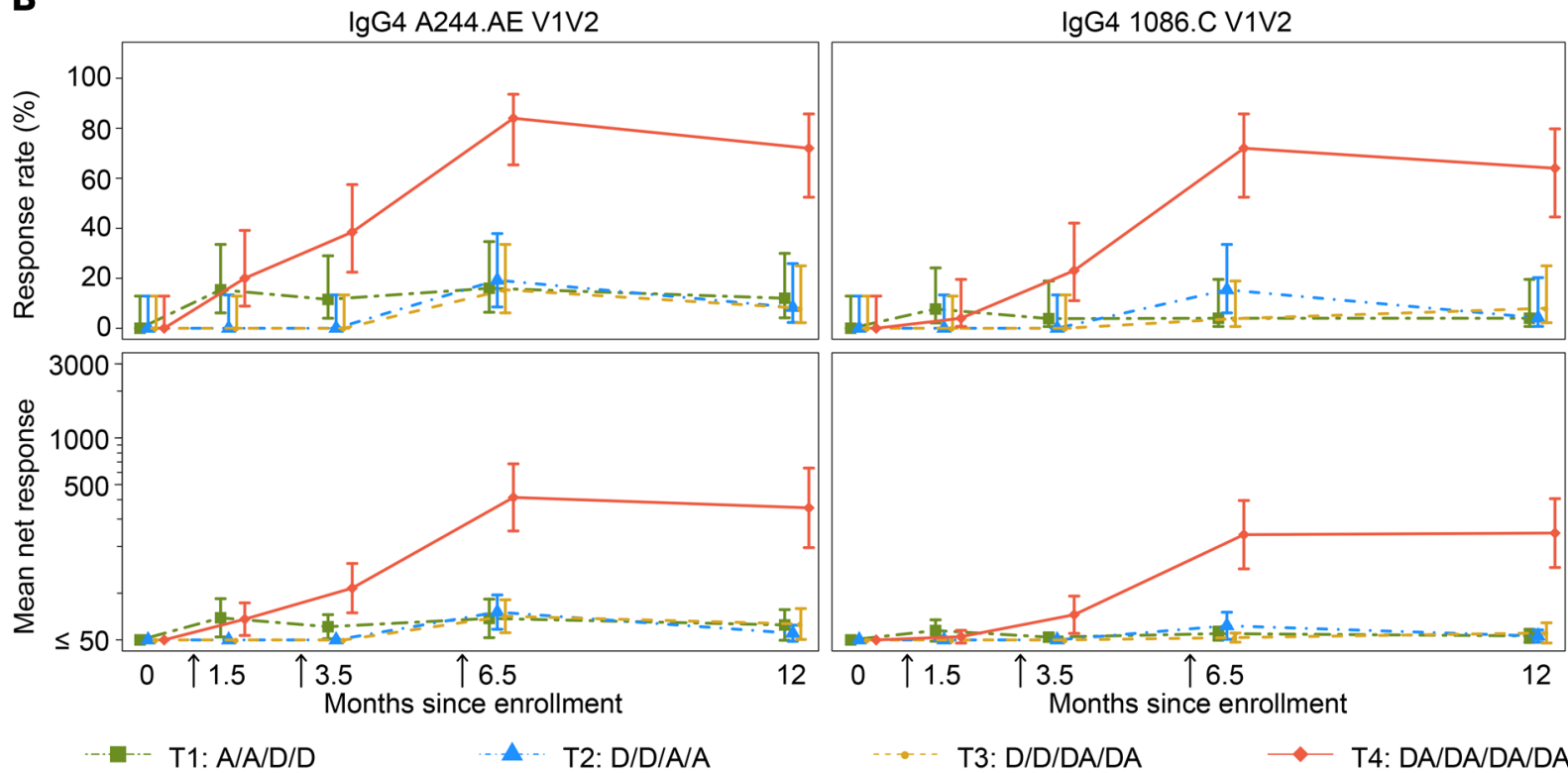

Figure 4. IgG3 and IgG4 binding-antibody responses in HVTN 105 participants over time, as measured by binding-antibody multiplex assay (BAMA) against 2 V1V2 antigens. Shown are the positive-response rates and 95\% Cl for IgG3 (A) and IgC4 (B) estimated using the score test method (top panels) and the geometric mean response magnitudes among all participants and $95 \%$ Cl based on an assumption of log(lgG) following a normal distribution (bottom panels) by time point and treatment group ( $n=25,26,26,25$ in T1-T4, respectively). The lines connect the response rates and geometric mean magnitudes between time points. Subtype AE V1V2: A244.AE V1V2; subtype C V1V2: 1086.C V1V2. Arrows indicate the second, third, and fourth vaccinations. D, DNA; A, AIDSVAX B/E.

T2: $P=0.0001)$. Similarly, T4 performed best in the neutralization magnitude-breadth (MB) analysis, with a significantly higher AUCMB than T2 and T3 ( $P=0.0004$ and 0.003, respectively) (Figure 7B). At month 12 (6 months after the fourth vaccination), nAb responses among all groups had declined; however, neutralization of MN.3.B was still present in groups $\mathrm{T} 2$, T3, and $\mathrm{T} 4$ (45.8\%, 46.2\%, and 60\%, respectively) (Figure 7C). Of note, the response to TH023.6.AE was detected at month 12 in T4 (64\%) and not in T2 and T3.

\section{Vaccine-induced cellular immunogenicity}

Positivity of responses for $\mathrm{CD}^{+} \mathrm{T}$ cells expressing IL-2 and/or IFN- $\gamma$ was determined 2 weeks after the second, third, and fourth vaccination time points and 6 months after the fourth vaccination. At 2 weeks after the fourth vaccination, the $\mathrm{CD} 4^{+} \mathrm{T}$ cell response rate to Env ranged from $36 \%$ to $60 \%$ among the 4 groups, with the highest response rate in $\mathrm{T} 3$, but the differences did not reach statistical significance (Figure 8A). At the month 12 time point (6 months after the fourth vaccination), $\mathrm{CD}^{+}$responses to Env declined to $17 \%-29 \%$ but again without significant differences between groups. $\mathrm{CD}^{+} \mathrm{T}$ cell responses to $\mathrm{Gag}$, which reflected immune responses to the DNA vaccine alone, were generally less frequent and of lower magnitude compared with Env (Figure 8B). Overall, there were no statistically significant differences in the rate or magnitude of $\mathrm{CD}^{+} \mathrm{T}$ cell responses to Gag between $\mathrm{T} 3$ and $\mathrm{T} 4$ at any 
A
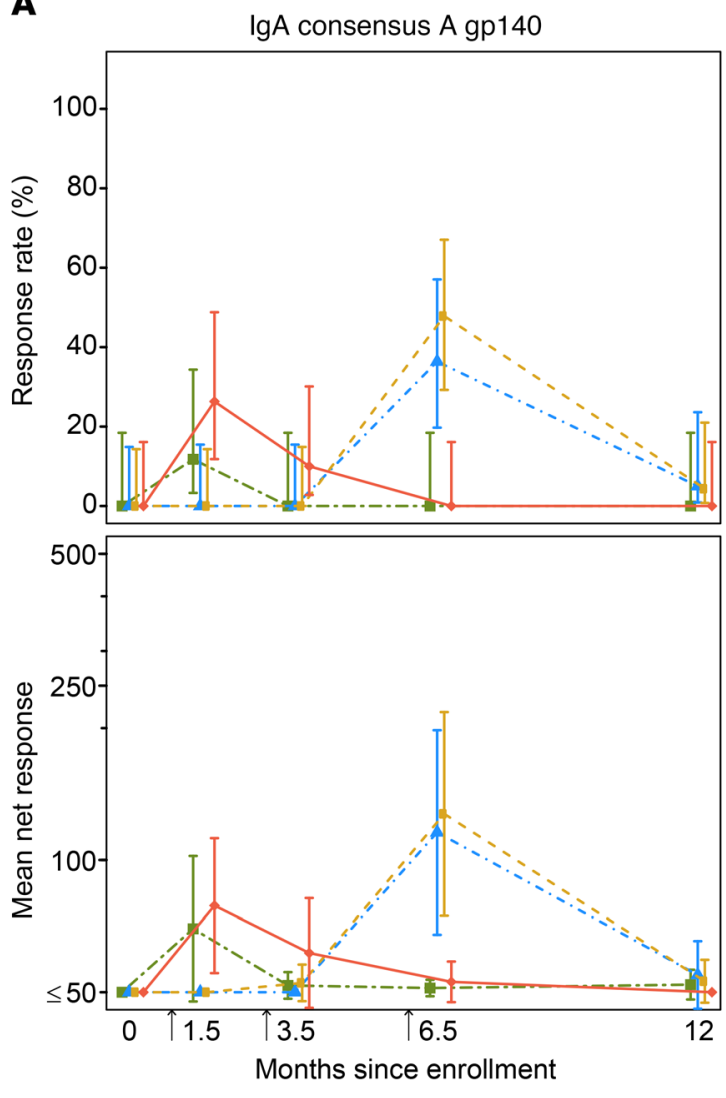

- $T 1: A / A / D / D$

- T2: D/D/A/A
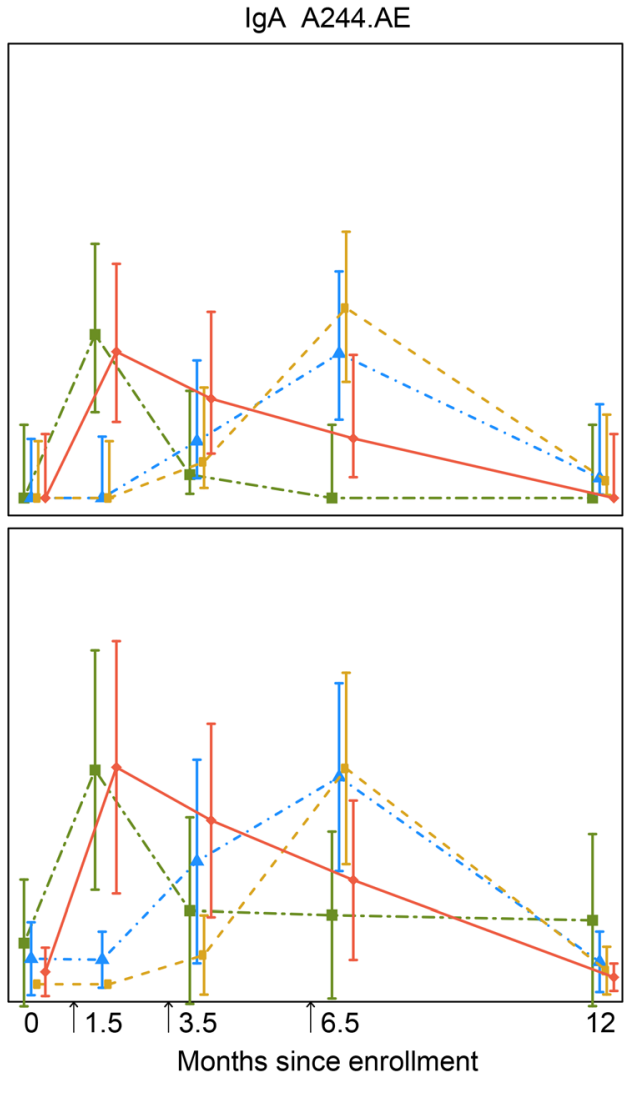

$\cdots$ T3: D/D/DA/DA $\rightarrow$ T4: DA/DA/DA/DA

\section{B}
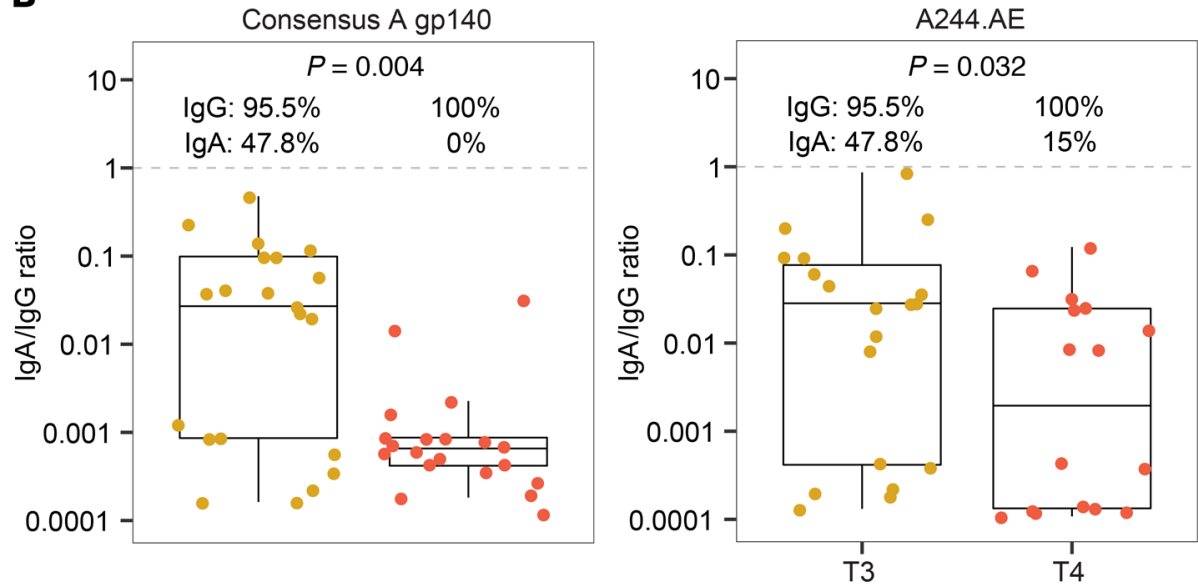

Figure 5. IgA binding-antibody responses in HVTN 105 participants over time and IgA/lgG ratio 2 weeks after the fourth vaccination, as measured by binding-antibody multiplex assay (BAMA) against consensus A gp140 and A244.AE antigens. (A) Shown are the positive-response rates and 95\% Cl estimated using the score test method (top panels) and the geometric mean response magnitudes among all participants and $95 \% \mathrm{Cl}$ based on an assumption of $\log (\operatorname{IgA})$ following a normal distribution (bottom panel) by time point and treatment group $(n=25,26,26,25$ in T1-T4, respectively). Arrows indicate the second, third, and fourth vaccinations. (B) The distribution and box-and-whisker plot of the IgA/lgG ratio in T3 and T4 (the midline of the box-and-whisker plot denotes the median and the ends of the box-and-whisker plot denote the 25 th and 75th percentiles). Shown at the top of plots are the percentages of positive responders to IgA and IgG, respectively, in T3 and T4; and the $P$ value is the testing difference in IgA/lgG ratio between T3 and T4 from Wilcoxon's rank-sum test. D, DNA; A, AIDSVAX B/E.

time point, although there was a trend for higher responses in $\mathrm{T} 3$. Finally, there were negligible $\mathrm{CD}^{+} \mathrm{T}$ cell responses to Env or Gag, as measured by IL-2 and IFN- $\gamma$, in any of the study groups (Supplemental Figure $4, \mathrm{~A}$ and $\mathrm{B})$.
$\mathrm{CD}^{+} \mathrm{T}$ cell polyfunctionality (PF) scores for Env (Figure 9A) and Gag (Figure 9C) peptides were assessed at the 4 time points using combinatorial PF analysis of single cells (COMPASS). Env-specific $\mathrm{CD} 4^{+} \mathrm{T}$ cell PF scores were similar across all 

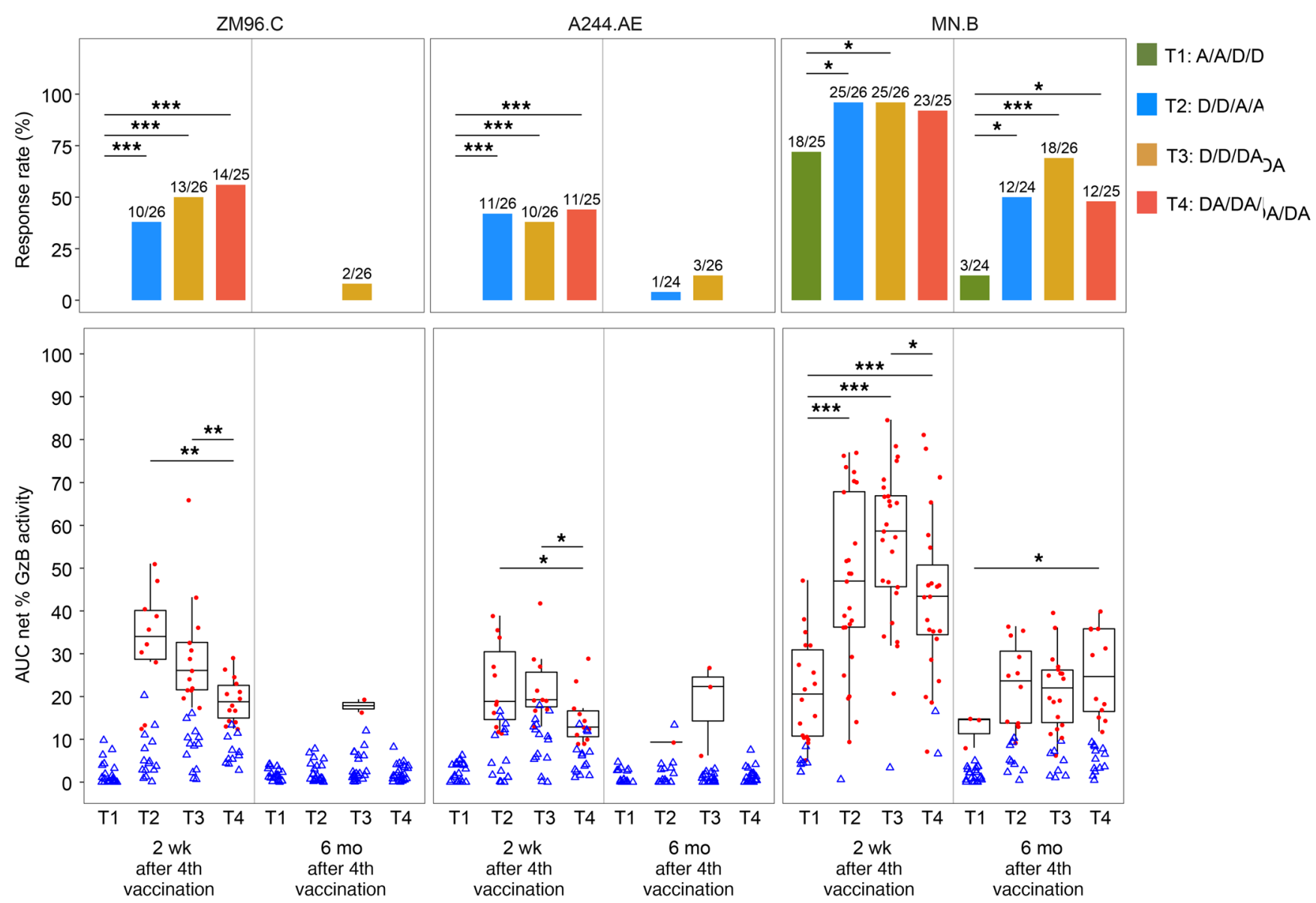

Figure 6. Antibody-dependent cell-mediated cytotoxicity (ADCC) at 2 weeks and 6 months after the fourth vaccination in HVTN 105. Shown are the response rates (top panels) and the distribution of AUC of granzyme B (GzB) activity (positive responders to peak granzyme B activity in red circles and nonresponders in blue triangles) and the box-and-whisker plots among the positive responders to peak granzyme $B$ activity (the midline of the box-andwhisker plot denotes the median and the ends of the box-and-whisker plot denote the 25th and 75th percentiles) (bottom panels) by time point and treatment group ( $n=25,26,26,25$ in T1-T4, respectively). Subtype C gp120: ZM96.C; subtype AE gp120: A244.AE; and subtype B gp120: MN.B. Bars and asterisks presented on the top of plots indicate the significant differences between treatment groups within the same visit $\left({ }^{*} P \leq 0.05 ;{ }^{* *} P \leq 0.01 ;{ }^{* *} P\right.$ $\leq 0.001$ ) without multiple-comparisons adjustment. The comparisons between treatment groups were performed using Fisher's exact test for response rates and Wilcoxon's rank-sum test for magnitudes. Fractions above bars on the top panels indicate numbers of positive responses over the total numbers of responses (positive and negative) by time point and treatment group. D, DNA; A, AIDSVAX B/E.

groups after the fourth vaccination, although somewhat lower for $\mathrm{T} 1$ and $\mathrm{T} 4$ (Figure 9A). PF scores for Gag again indicated that at least 2 doses of DNA were required for optimal responses, and responses in $\mathrm{T} 3$ and $\mathrm{T} 4$ peaked 2 weeks after the third vaccination, with the PF scores for T3 significantly higher than for T4 at that time point $(P=0.007)$, as well as at 6 months after the fourth vaccination $(P=0.005)$.

There were 2 dominant polyfunctional populations among Env-specific $\mathrm{CD}^{+} \mathrm{T}$ cell responses, one identified by 4 markers $\left(\mathrm{IFN}-\gamma^{+} \mathrm{IL}-2^{+} \mathrm{TNF}-\alpha^{+} \mathrm{CD} 4 \mathrm{OL}^{+}\right)$and the second with 3 markers (IL-2 ${ }^{+} \mathrm{TNF}-\alpha^{+} \mathrm{CD} 4 \mathrm{OL}^{+}$but without IFN- $\gamma$ ) (Figure 9B). A high mean probability of response for both of these populations across all groups at most time points indicates that many, and often most, individuals had detectable $\mathrm{CD} 4^{+} \mathrm{T}$ cells expressing these markers above the level in the negative control stimulation. Cells expressing only IL-2 and IL-2 in combination with CD4OL were detected in some individuals, and there were minimal differences between groups. The other commonly expressed subsets included cells coexpressing TNF- $\alpha$ and CD40L, with or without IFN- $\gamma$. Compared with Env, the same two 3- and 4-function subsets were dominant for Gag (Figure 9D), with highest expression of these subsets in T3, likely accounting for the significantly higher PF score compared with the other groups even though T3 only received 2 doses of Gag compared with the 4 doses that $\mathrm{T} 4$ received. A few of the other double- and triple-positive cells observed for Env were also detected in more individuals in T3 for Gag. These results highlight the induction of highly polyfunctional Env- and Gagspecific $\mathrm{CD}^{+} \mathrm{T}$ cells, and are also in agreement with the highest IFN- $\gamma$ and/or IL-2 response rates for T3 (although not statistically significant) compared with the other groups.

\section{Overview of the induced immune responses}

A global representation of the distribution of the cellular and humoral immunogenicity by response rate is shown in Figure 10. The analysis indicates that coadministration of DNA and 
A
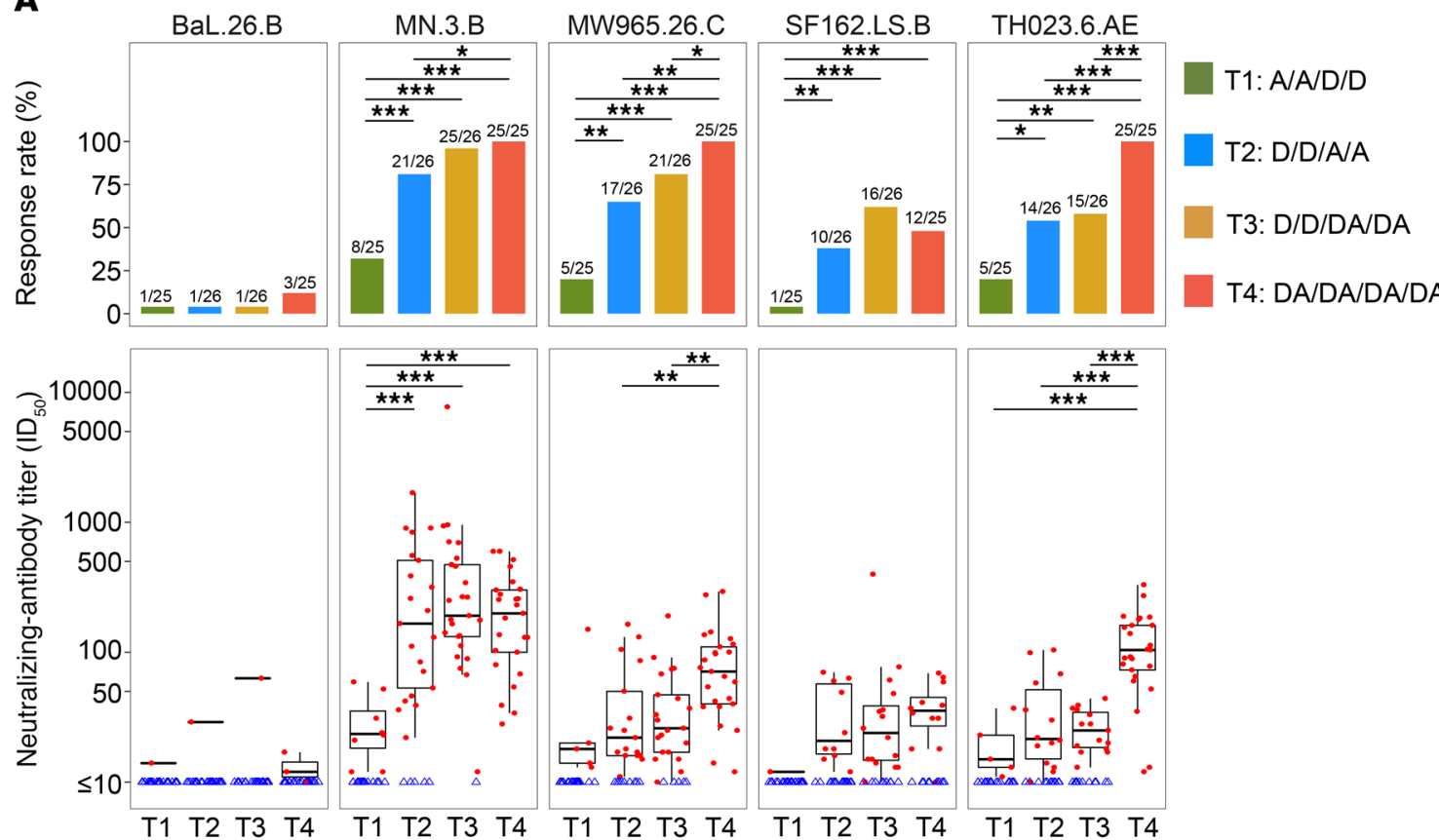

B

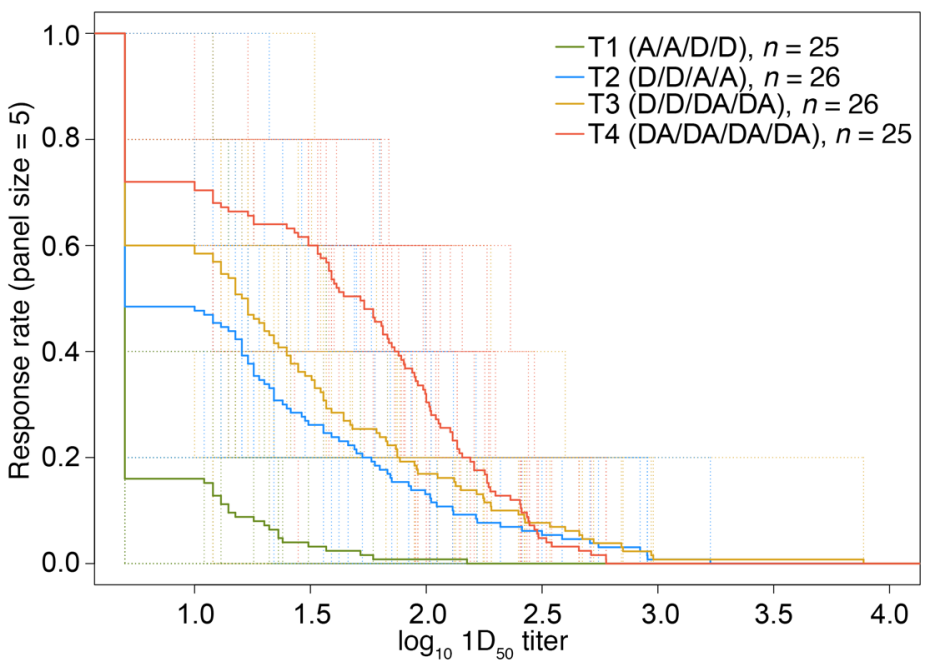

C

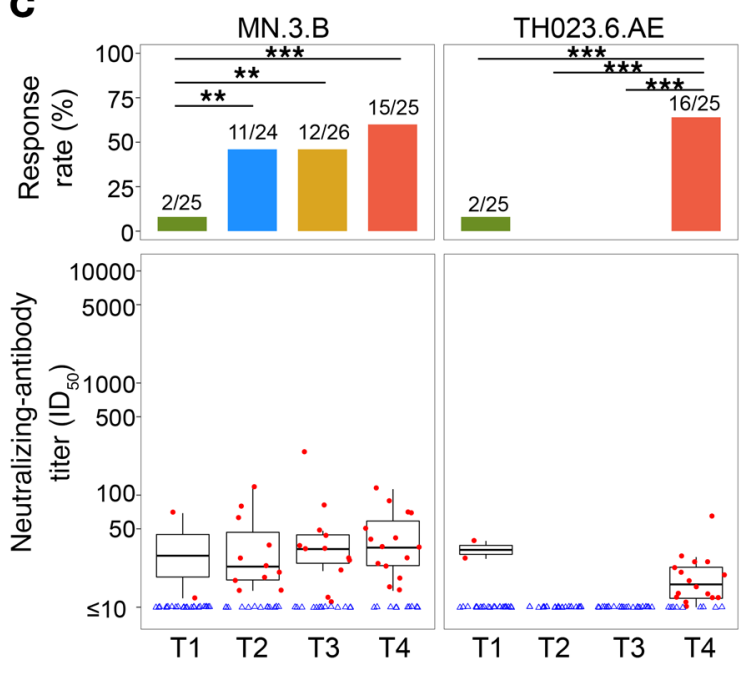

Figure 7. Neutralizing-antibody responses in HVTN 105. (A) Tier 1 Env-pseudotyped viruses (BaL.26.B, MN.3.B, MW965.26.C, SF162.LS.B, and TH023.6.AE) were tested in the TZM-bl neutralization assay 2 weeks after the fourth vaccination (peak time point). Bar plots show positive-response rates by treatment group on top panels. The bottom panels show the distribution of response titer (positive responses in filled red circles and negative responses in open blue triangles) and the box-and-whisker plots of response titer among positive responders (the midline of the box-and-whisker plot denotes the median and the ends of the box-and-whisker plot denote the 25th and 75th percentiles) by treatment group $(n=25,26,26,25$ in T1-T4, respectively). (B) Neutralizing-antibody magnitude breadth (AUC-MB) curves for TZM-bl based on all 5 isolates. (C) Neutralizing-antibody responses to MN.3.B and TH023.6.AE at 6 months after the fourth vaccination. Bars and asterisks presented on the top of plots in $\mathbf{A}$ and $\mathbf{C}$ indicate the significant differences between treatment groups within the same visit $\left({ }^{*} P \leq 0.05 ;{ }^{* *} P \leq 0.01\right.$; ${ }^{* * *} P \leq 0.001$ ) without multiple-comparisons adjustment. The comparisons between treatment groups were performed using Fisher's exact test for response rates and Wilcoxon's rank-sum test for magnitudes. The comparisons of AUC-MB at peak time point in B between treatment groups from Wilcoxon's rank-sum test show that AUC-MB in T4 is significantly higher than T1-T3, with $P<0.001, P<0.001$, and $P$ $<0.003$, respectively, and AUC-MB in T1 is significantly lower than T2-T3, with $P<0.001$. Fractions above bars in $\mathbf{A}$ and $\mathbf{C}$ indicate the numbers of positive responders over the total number of responses (positive and negative). D, DNA; A, AIDSVAX B/E.

protein ( $\mathrm{T} 3$ and $\mathrm{T} 4$ groups) can improve the response rates for most of the immune responses measured and induce a favorable immune profile at later time points. Suboptimal responses were seen when protein served as a prime for subsequent boosting with DNA (T1), which was particularly evident at the month 12 time point (6 months after the fourth vaccination). While priming with DNA and boosting with protein alone (T2) was immunogenic, the response was less favorable than the coadministration groups for IgA, IgG3, IgG4, and $\mathrm{CD} 4^{+} \mathrm{Gag}$ responses at month 6.5 ( 2 weeks after the fourth vaccination), 
A

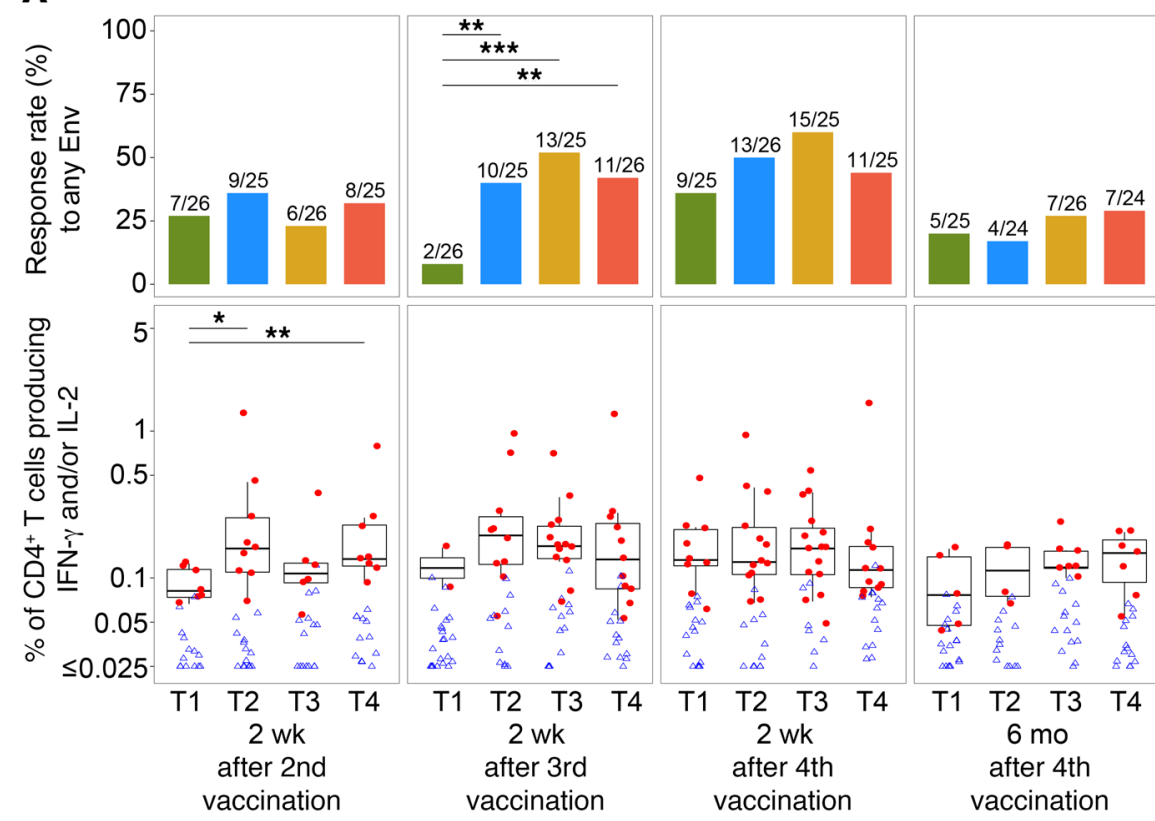

B

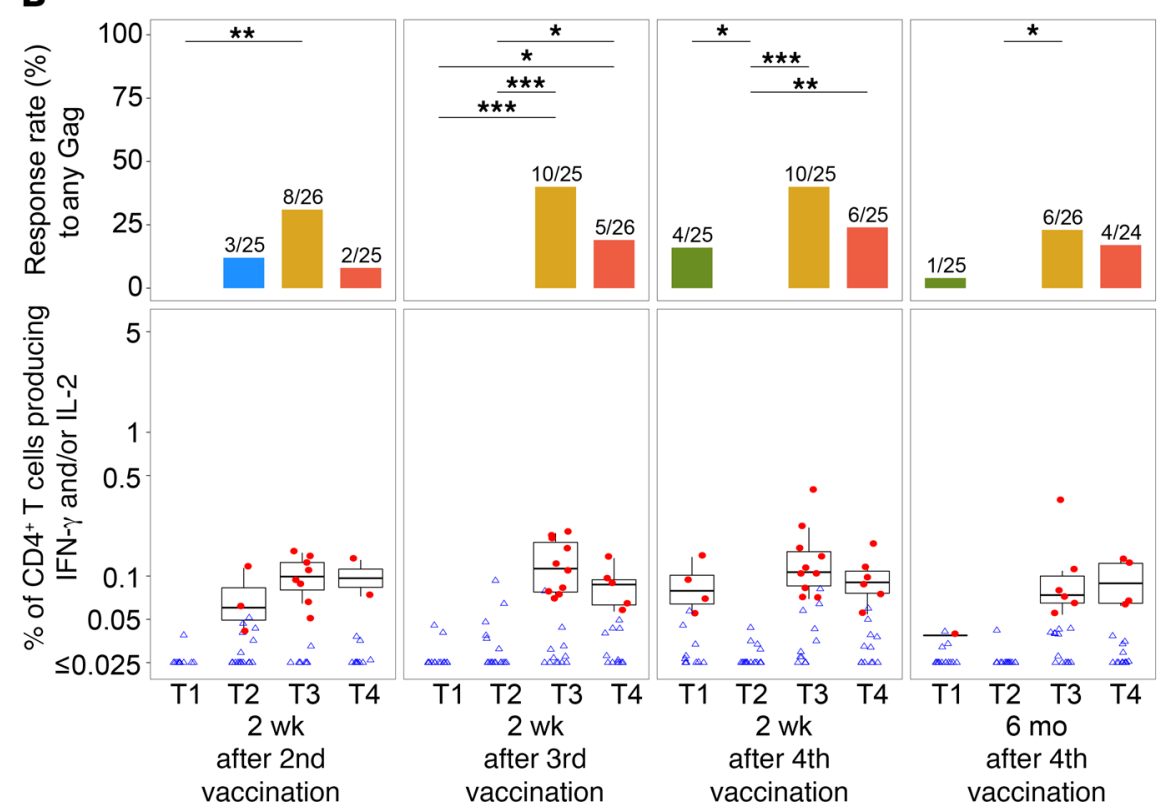

$T 1: A / A / D / D$

T2: D/D/A/A

T3: D/D/DA/DA

T4: DA/DA/DA/DA

Figure 8. $\mathrm{CD4}^{+} \mathrm{T}$ cell responses elicited in HVTN 105 , as measured by intracellular cytokine staining (ICS), and reported as the percentage of cells producing IFN- $\gamma$ and/or IL-2 in each treatment group. (A) CD4 ${ }^{+} \mathrm{T}$ cell responses to any HIV Env peptide pools (Any Env), all vaccine-matched: ZM96 gp140-Env1, ZM96 gp140-Env2, and 92TH023-Env. (B) CD4+ $\mathrm{T}$ cell responses to HIV Gag peptide pool (Any Gag): ZM96 Gag. Bar plots on top panels show the positive-response rates by time point and treatment group ( $n=25,26,25,25$ in T1-T4, respectiveIy). The bottom panels show the distribution of response magnitudes (positive responses in filled red circles and negative responses in open blue triangles) and the box-and-whisker plots of magnitudes among the positive responders (the midline of the box-and-whisker plot denotes the median and the ends of the box-and-whisker plot denote the 25 th and 75 th percentiles). Fractions above bars on top panels indicate the numbers of positive responders over the total numbers of responses (positive and negative). Bars and asterisks on top of plots indicate the significant differences between treatment groups $\left({ }^{*} P \leq 0.05 ;{ }^{* *} P \leq 0.01 ;{ }^{* *} P \leq\right.$ 0.001 ) without multiple-comparisons adjustment. The comparisons between treatment groups were performed using Fisher's exact test for response rates and Wilcoxon's rank-sum test for magnitudes. D, DNA; A, AIDSVAX B/E. and for ADCC, nAb, IgG4, and CD4 Gag at month 12 (6 months after the fourth vaccination).

\section{Vaccine-induced sero-reactivity was uncommon}

Vaccine-induced sero-reactivity (VISR) was assessed by 4 licensed diagnostic kits used to detect HIV antibodies at 4 time points during the study. VISR was detected by all of the 4 assays in $4 \%-122 \%$ of participants in each group ( 3 participants in T4 and in 1 participant in each of the other 3 groups). None of the participants in the study became infected with HIV (Supplemental Table 2).

\section{Discussion}

This trial evaluated different combinations of DNA (DNA-HIVPT123) and protein (AIDSVAX B/E) vaccinations to determine which strategy would best elicit favorable HIV-specific antibody and $\mathrm{T}$ cell responses, as defined by the correlates of risk identified in RV144, over the 12-month duration of protocol follow-up. Our study showed that coadministration of DNA and protein from the initial vaccination (T4) leads to induction of potentially protective anti-HIV antibody responses within the first 6 weeks, as opposed to the DNA prime-protein boost regimen reflecting the RV144 vaccination schedule (T3), which induces similar responses, but not until after the final boosts at month 6. Otherwise, there were no significant differences between the early and delayed coadministration groups (T4 vs. T3) for induction of any of the currently known RV144 potential correlates of HIV acquisition risk. Of note, we observed a significant increase in HIV-specific IgG4 at the month 12 time point in the early coadministration group 
A

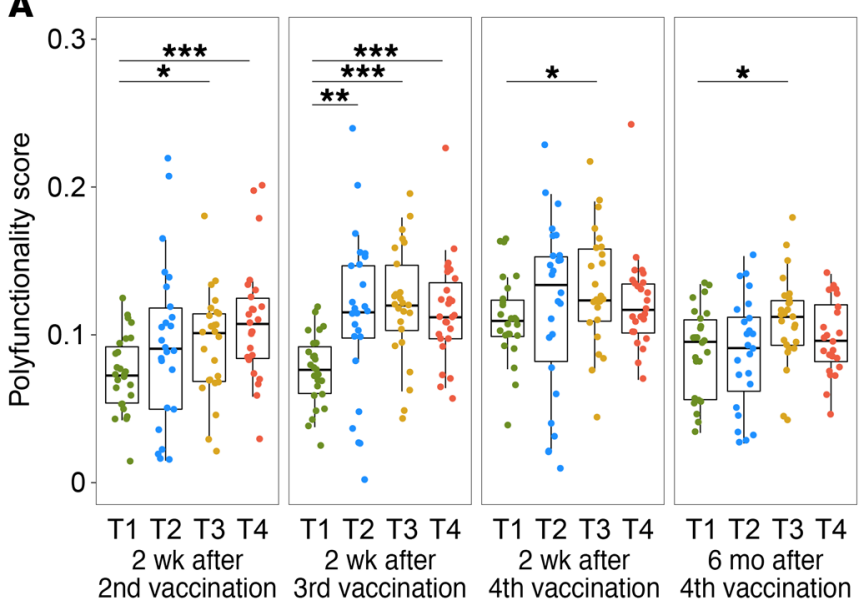

C

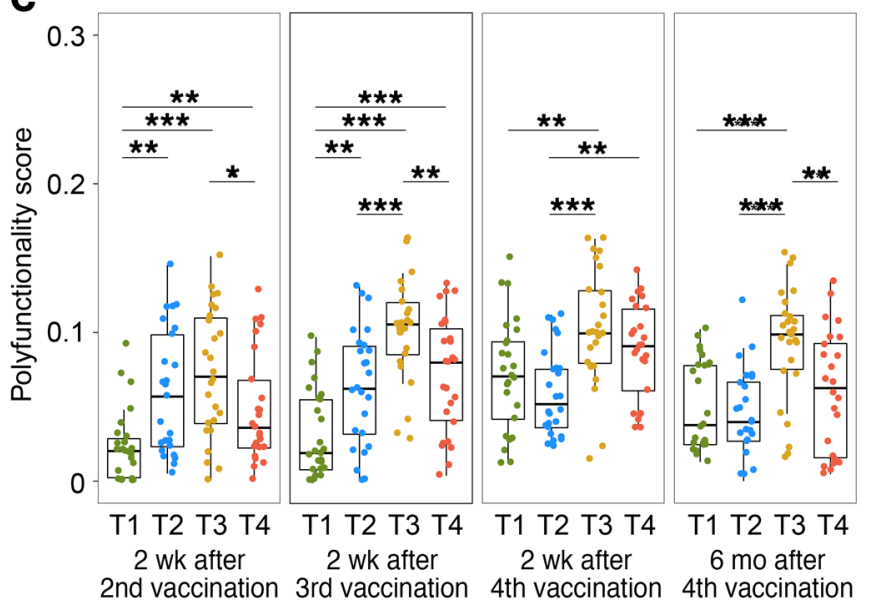

B

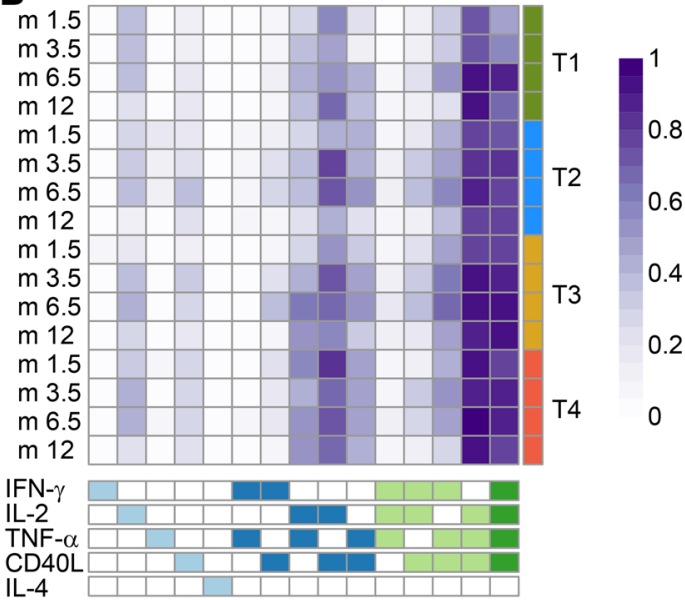

D

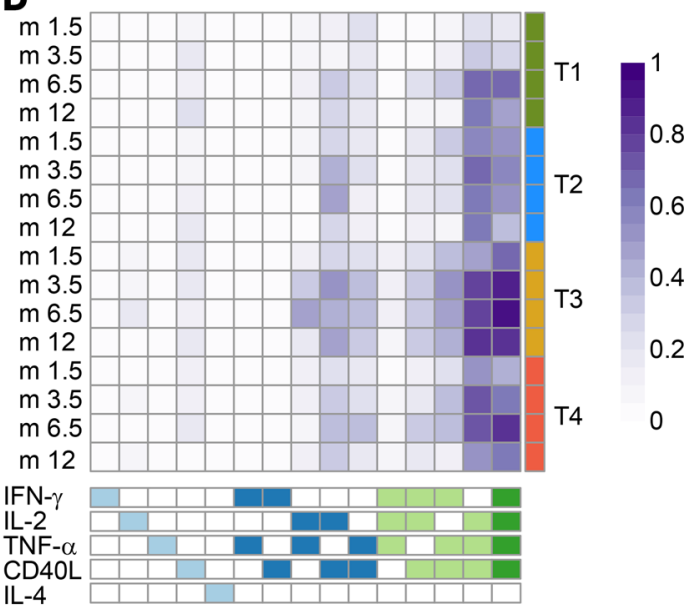

Figure 9. COMPASS CD4 ${ }^{+} T$ cell polyfunctionality (PF) scores and mean probability of response heatmaps. The distribution and the box-and-whisker plot of PF scores for all vaccine-matched HIV Env peptide pools: ZM96 gp140-Env1, ZM96 gp140-Env2, and 92TH023-Env (A) and for ZM96 Gag peptide pool (C) by time point and treatment group ( $n=25,26,25,25$ in T1-T4, respectively). The midline of the box-and-whisker plot denotes the median and the ends of the box-and-whisker plot denote the 25th and 75 th percentiles. Bars and asterisks on top of box-and-whisker plots indicate the significant differences between treatment groups using Wilcoxon's rank-sum test $\left({ }^{*} P \leq 0.05 ;{ }^{* *} P \leq 0.01\right.$; ${ }^{* * *} P \leq 0.001$ ) without multiple-comparisons adjustment. Heatmaps for $\mathrm{CD}^{+} \mathrm{T}$ cell response to any Env (B) and ZM96 Gag (D) show the mean posterior probabilities of antigen-specific responses from COMPASS. Columns correspond to the different subsets of cytokines being considered and rows correspond to mean across the individual participants in each treatment group at each time point. Each cell shows the probability that the corresponding antigen-specific subset (column) is being expressed in the corresponding treatment group in average (row), and is color coded ranging from white (zero) to dark purple (one).

(T4), although the functional significance of HIV-specific IgG4 in the context of conferred protection from infection is currently unclear and subsequently discussed herein. The regimen with DNA priming administered at month 0 and 1 with AIDSVAX $\mathrm{B} / \mathrm{E}$ boosting alone at months 3 and 6 (T2) induced immune responses similar to those of T3, but 4 doses of DNA (comparing T3 vs. T2) were superior in terms of inducing a better $\mathrm{CD}^{+} \mathrm{T}$ cell responses to Gag, which was contained only in the DNA candidate. Finally, priming with protein and boosting with DNA (T1) clearly led to inferior immunologic results compared with the other groups at the month 6 time point and beyond.

In this proof-of-concept study, we demonstrated that substituting DNA for the ALVAC vector (T3) is a suitable alternative. Interestingly, the prime-boost regimen in our study was clade mismatched relative to RV144 (DNA plasmid expressed a subtype C Env rather than the clade-matched AE Env in RV144), raising the possibility that subtype C Env can serve as a universal prime for heterologous Env protein boosts. In fact, comparing data from this group to $\operatorname{RV} 144(5,20)$, the peak BAMA IgG response rate 2 weeks and 6 months after the final vaccination (months 6.5 and 12) was $96 \%-100 \%$ and $48 \%-81 \%$, respectively, against the 2 strains (subtype B MN gp120 and subtype A/E A244 gp120) contained in AIDSVAX B/E, the vaccine common to both HVTN 105 and RV144. Similarly, IgG responses to the V1V2 scaffold of subtype C 1086.C V1V2, which was identified as an inverse correlate of risk in RV144, were present at months 6.5 and 12 in $100 \%$ and $67 \%$ of participants in T3, respectively. Of note, the magnitude of the IgG response to the V1V2 scaffold was quite high in T3 (peak geometric mean titer [GMT] to 1086.C V1V2 in T3 was 15,002 [95\% CI: 10,099-22,285]). Importantly, responses in our study were more durable than in RV144, persisting at month 12 (6 months after the fourth vaccination) in up to $40 \%$ of $\mathrm{T} 3$ recipients versus $10 \%$ in 
A

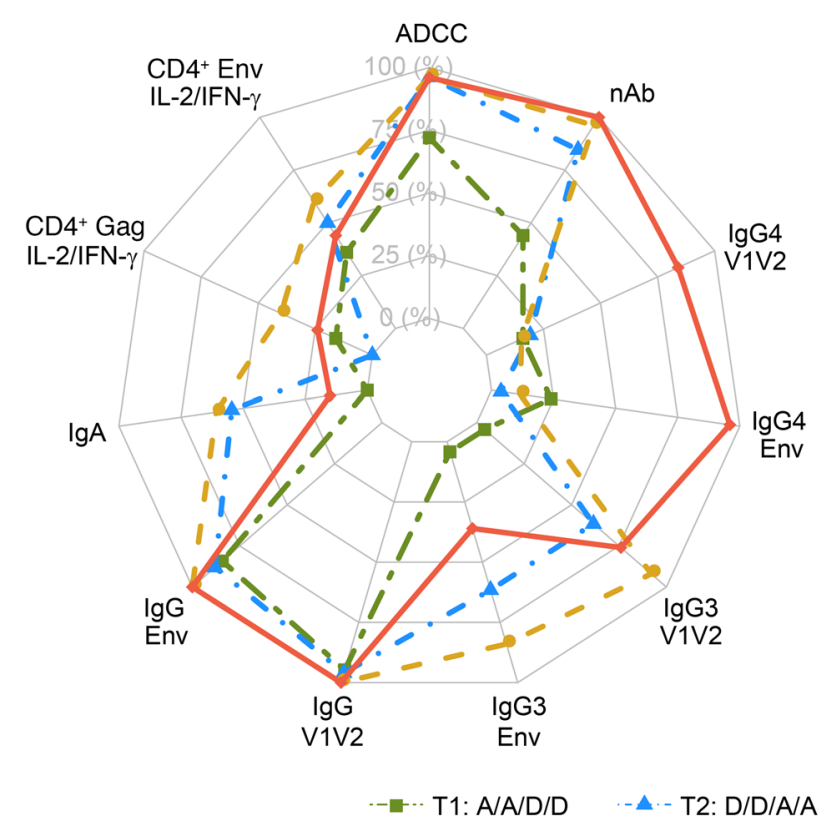

B

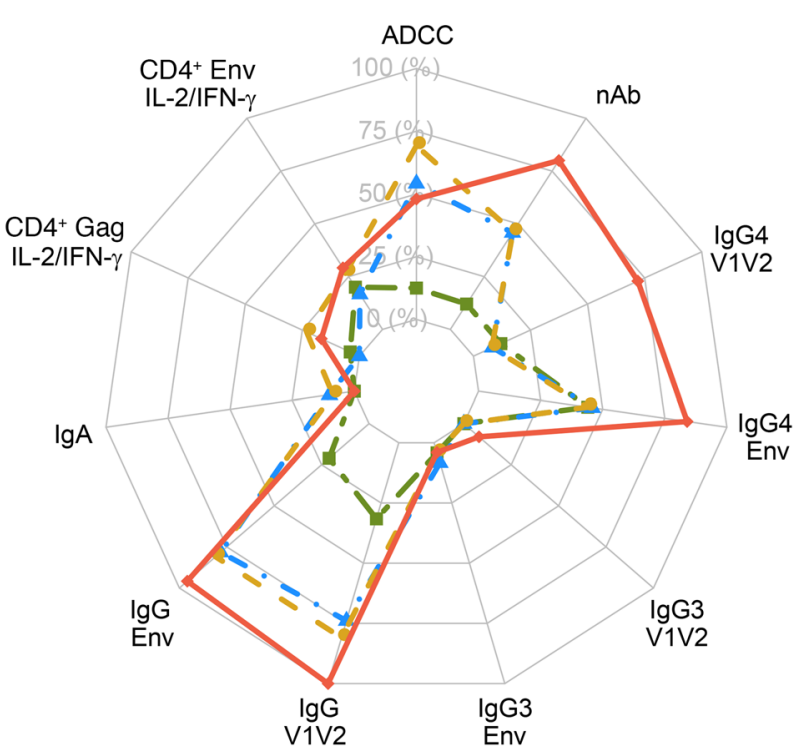

- T3: D/D/DA/DA $\longrightarrow$ T4: DA/DA/DA/DA

Figure 10. Radar plots. Maximum response rates of each assay readout at 2 weeks (A) and 6 months (B) after the fourth vaccination. Antigens included in each assay readout are as follows: For IgC binding-antibody responses to Env, antigens include A244.AE, MN.B, ZM96.C, Con S gp140 CFI, as well as Con 6 gp120. For IgG binding-antibody responses to V1V2, antigens include 1086.C V1V2, CaseA2_gp70_V1V2.B, CaseA2_V1/V2/169K.B, A244.AE V1V2, as well as ZM96.C V1V2. For IgA binding-antibody responses, antigens include consensus A gp140 and A244.AE. For neutralizing-antibody (nAb) responses, antigens include BaL.26.B, MN.3.B, MW965.26.C, SF162.LS.B, and TH023.6.AE. For antibody-dependent cell-mediated cytotoxicity (ADCC) responses, antigens include ZM96.C, A244.AE, and MN.B. For CD4+ T cell intracellular cytokine staining, Env antigens are ZM96 or $92 \mathrm{TH023}$ peptide pools (Any Env), and the Gag antigen is ZM96 peptide pool (Any Gag). D, DNA; A, AIDSVAX B/E.

RV144 vaccine recipients to 1086.C V1V2 antigen, although an even greater response at month 12 would be desirable. By extension, the coadministration of DNA and protein at all vaccination time points (T4) also compares favorably to RV144. This is especially evident when considering the rapid induction of antibodies, but also in terms of sustained responses. All participants in T4 had remarkably elevated IgG response to $1086 . \mathrm{C} \mathrm{V1V} 2$ antigen $(100 \%$ response rate and $\mathrm{GMT}=14,809$ at 6.5 months), with $71 \%$ of vaccinees having detectable antibody response at 12 months from first vaccination.

In RV144, IgG3 responses against V1V2 were associated with low risk of HIV-1 acquisition, and postulated to mediate protective HIV-specific antibody Fc effector functions such as ADCC (7) and antibody-dependent cell-mediated phagocytosis $(21,22)$. There is also evidence from experiments with monoclonal antibodies derived from B cells of RV144 vaccinees that HIV-1 Env IgA blocks ADCC-mediating antibodies, consistent with the findings that ADCC correlated with decreased risk of HIV-1 infection when Env IgA was low and that an elevated IgA/IgG ratio correlated with HIV-1 risk (8). In our study, high peak net percentage granzyme B ADCC activity and response rates were noted for groups T2, $\mathrm{T} 3$, and T4 against subtype B gp120 and remained elevated at the month 12 time point (6 months after the fourth vaccination). As seen in RV144 (20), the IgG3 responses in HVTN 105 significantly declined over time, although the persisting ADCC activity at month 12 could be explained either by the favorable low IgA/IgG ratio at the completion of vaccine regimen dosing (especially seen in T4) and/or by the overall persistence of other IgG subclasses such as IgG1 or alteration of IgG1 glycosylation mediating ADCC at the durability time point, as demonstrated through a systems serology approach (23).

In our trial, vaccination with 4 protein immunizations (T4) elicited greater Env-specific IgG4 responses than the other immunization groups, although this is more likely explained by the administration of the AIDSVAX B/E protein at 4 time points over the 6-month vaccination schedule as opposed to coadministration with DNA, per se. Bias toward the induction of the HIVspecific IgG4 subclass has been seen in other studies of repeated vaccination with AIDSVAX B/E $(24,25)$, including in VAX003, which administered AIDSVAX $\mathrm{B} / \mathrm{E}$ alone at 7 time points to injection-drug users over a 3-year follow-up (3), and in RV307, which administered 2 additional doses of AIDVAX B/E to RV144 participants 6-8 years after previous vaccination (26). This phenomenon has also been noted with acellular pertussis vaccine after repeated vaccination in a pediatric population. However, in a follow-up study in children infected with Bordetella pertussis, the IgG-subclass distribution did not differ from healthy vaccinated children, suggesting the absence of clinical importance (27). Therefore, the significance of high levels of durable Env-specific IgG4 and its correlation with existing in vitro ADCC assays in the setting of HIV acquisition is unclear and should be further examined in ongoing HIV vaccine efficacy trials.

The $\mathrm{CD}^{+} \mathrm{T}$ cell responses were similar across all the groups after the fourth vaccination. This is especially surprising for Gag since that was only encoded for in the DNA, and this difference in response rates may indicate some interference or antigenic compe- 
tition due to the additional doses of Env protein. Consistent with the IL-2 and/or IFN- $\gamma$ response rates, the Gag-specific PF scores were also lower for the group with 4 protein vaccinations. Overall, CD $4^{+}$ $\mathrm{T}$ cell responses included multiple 2-, 3-, and 4-function subsets, mainly including CD4OL as one of the functions. This suggests that a major function for these vaccine-induced $\mathrm{T}$ cells is to provide help to B cells. It may be surprising that IL-4 was not detected among these responding cells, and this could be due to low sensitivity for detection of IL-4 in our assay or in fact due to poor induction of $\mathrm{T}$ helper type 2 (Th2) cells that may be necessary for a more durable antibody response. It would be potentially of benefit to also induce $\mathrm{CD}^{+} \mathrm{T}$ cells in any candidate HIV regimen. Although the regimens we tested did not induce CD8 ${ }^{+} \mathrm{T}$ cells, alternate DNA vaccines, especially when administered with electroporation, are now capable of inducing $\mathrm{CD}^{+} \mathrm{T}$ cells and may be appropriate substitutions for the DNA part of the vaccine regimen (28-30).

The type of prime as well as the combination and sequence in which vaccines are administered influence the quality of immune responses. Administering a protein-only prime (T1) did not elicit strong, durable antibody responses in this study. It is possible that with a more potent adjuvant, as was used in the preclinical studies in which a protein prime did elicit strong responses, different results may be observed. The group with DNA-only prime followed by protein-only boost (T2) did elicit antibody responses, but these were generally lower in response rate and magnitude than the groups that included coadministration of DNA and protein. The 2 groups that received DNA and protein coadministration at the last 2 (T3) or at all 4 (T4) vaccination time points elicited not only high V1V2 IgG and antibody effector function (ADCC) responses, but also tier $1 \mathrm{nAb}$ and $\mathrm{CD} 4^{+} \mathrm{T}$ cell responses, higher or similar to those observed in RV144. Based on our data, it is not possible to make a firm conclusion about whether $\mathrm{T} 3$ versus T4 is the preferred regimen, as the potential correlates of risk identified in RV144 have not yet been confirmed. T4 induced early responses requiring, however, more vaccine doses. Both T3 and $\mathrm{T} 4$ induced equivalent cellular and humoral responses, with the exception of a marked increase in IgG4 with subsequent protein boost in T4; the physiologic significance of that increase is yet to be determined. Future studies will examine other potential correlates of protective immunity, such as avidity of IgG antibodies and other antibody Fc effector functions.

While no DNA vaccine is licensed for use in humans, many DNA vaccines are approved for veterinary applications. DNA vaccines are currently widely evaluated in clinical trials against many infectious diseases $(31,32)$ and for HIV prevention and therapeutics. Ongoing trials are testing the protein and DNA combination regimens with alternative adjuvants given with envelope protein (e.g., MF59 or AS01) (HVTN 108 [NCT02915016] and HVTN 111 [NCT02997969]) (33), modification of the schedule of envelope protein administration, or coadministration with other vaccine platforms such as adenoviral vector 26 or MVA. There are also other approaches to optimize DNA priming, such as formulation with cytokine adjuvants or administration by intramuscular or intradermal electroporation, that can also be examined to expand our understanding of tailoring an optimal immunologic signature to prevent acquisition of HIV. Current studies are testing ALVAC as an HIV vaccine candidate including a pivotal phase $2 \mathrm{~b} / 3$ trial in
South Africa (HVTN 702 [NCT02968849]) in addition to earlyphase studies (HVTN 107 [NCT NCT03284710] and HVTN 120 [NCT03122223]). DNA vaccines are safe, easy to manufacture, and have great molecular stability and flexibility with immunogen design. Based on our study, DNA provides a suitable alternative platform to ALVAC; however, a larger efficacy study is needed to confirm the findings of our proof of concept.

In conclusion, DNA appears to be a good alternative to viral vectors, and its early coadministration with protein can rapidly induce a potentially protective antibody response that would enhance its value to public health.

\section{Methods}

Trial design. The objectives of the study were to evaluate the safety and tolerability (primary objective) and the immunogenicity (secondary objective) of the combination of AIDSVAX B/E and DNA-HIVPT123 administered in different sequences or simultaneously in HIVuninfected healthy adults.

Participants were screened at 7 HVTN sites in 6 US cities: Nashville, Tennessee; New York, New York; Rochester, New York; Philadelphia, Pennsylvania; Seattle, Washington; and San Francisco, California. Participants were males and nonpregnant females who met the inclusion criteria as listed on https://clinicaltrials.gov. Women of child-bearing potential were advised to avoid pregnancy for 12 months after enrollment. All participants were assessed to be at low risk for HIV acquisition, were counseled routinely about HIV risk reduction, and were assessed for potential social impacts of study participation. Figure 1 shows a CONSORT statement flow chart of study enrollment, allocation, and safety analysis.

Participants were randomly assigned to 1 of 4 groups with an allocation ratio of 1:1:1:1 (Table 1). Participants received different combinations of AIDSVAX B/E, DNA-HIV-PT123, and placebo, administered intramuscularly. AIDSVAX B/E consisted of $300 \mu \mathrm{g}$ of subtype B (MN) HIV gp120 glycoprotein and $300 \mu$ g of subtype A/E (A244) HIV gp120 glycoprotein adsorbed onto aluminum hydroxide gel adjuvant and administered into the right deltoid muscle. DNA-HIV-PT123 contained a mixture of 3 DNA plasmids: (a) clade C ZM96 gag, (b) clade C ZM96 gp140, and (c) clade C CN54 pol-nef, delivered at a total dose of $4 \mathrm{mg}$ administered into the left deltoid muscle via needle and syringe. The placebo consisted of sodium chloride for injection, 0.9\% USP. The randomization sequence was obtained by computer-generated random numbers and provided to the site by a central data monitoring center. A pharmacist at each site was responsible for ensuring the security of the randomization code and maintaining the anonymity of the sample by covering the syringe containing the study products. All participants and study staff, apart from the study pharmacist, were blinded to treatment assignment.

Safety assessment. Reactogenicity assessments were performed on all participants for 3 days following each injection. Participants recorded symptoms using a postvaccination symptom log and were contacted daily by the study site during each reactogenicity assessment period. Participants returned to the clinic 2 weeks after each vaccination and 9 months after the first vaccination for clinical evaluation for local and systemic signs and symptoms and laboratory testing of hematologic, renal, and hepatic analytes. These safety evaluations were coded according to the Medical Dictionary for Regulatory Activities terminology, and graded using the US Divi- 
sion of AIDS Table for Grading the Severity of Adult and Pediatric Adverse Events $(34,35)$. AEs were assessed for their relationship to the study product. Reactogenicity symptoms among the treatment groups were tabulated by severity.

Sample processing. Serum for humoral assays was obtained from serum-separating tubes (SSTs) and frozen at $-80^{\circ} \mathrm{C}$. Peripheral blood mononuclear cells (PBMCs) for cellular assays were isolated and cryopreserved from heparin-anticoagulated whole blood within 6 hours of venipuncture, as described previously (36).

Binding-antibody assays. Serum HIV-1-specific IgG, IgG3, IgG4, and IgA responses were measured with a custom HIV-1-binding antibody multiplex assay (BAMA) as previously described $(7,37)$ using gp120 proteins and V1V2 antigens detailed previously (38). Details of the antigens included are listed in Supplemental Table 3.

ADCC. ADCC-mediated antibody responses were measured by ADCC GranToxiLux (GTL) and tested against ZM96.C, A244.AE, and MN.B using gp120-coated cells (percentage granzyme B readout) (see Supplemental Table 3 for additional details on the antigens used). Participant sera were incubated with effector cells and gp120-coated target cells (39) and ADCC was quantified as net percentage granzyme B activity, which is the percentage of target cells positive for GTL detected by flow cytometry. For each participant at each time point, the percentage granzyme B activity was measured at 6 dilution levels: 1:50, 1:250, 1:1250, 1:6250, 1:31,250, and 1:156,250 for each antigen. For some participants, percentage granzyme B activity was measured at an additional 6-dilution series: 1:6250, 1:31,250, $1: 156,250,1: 781,250,1: 3,906,250$, and 1:19,531,250 for some antigens. The additional dilution series was used because the endpoint dilution for some of the samples could not be determined with the initial series. Overlapping dilutions between series were averaged. The analyses in the data focused on the following readouts: (a) peak net percentage granzyme B activity defined as the maximum activity across the 6 or 9 dilution levels (peak activity), and (b) nonparametric area under the net percentage granzyme B activity versus $\log _{10}$ (dilution) curve (AUC), calculated using the trapezoidal rule. Peak activity less than $0 \%$ was set to $0 \%$. A positive response was defined as peak activity greater than or equal to $8 \%$.

$H I V-1-$ specific $n A b$ assays. nAbs against HIV-1 were measured as a function of reductions in Tat-regulated luciferase (Luc) reporter gene expression in TZM-bl cells 2 weeks and 6 months after the last vaccination (40). The assay measured neutralization titers against 2 Env-pseudotyped viruses that exhibit a tier 1 neutralization phenotype and are matched to the vaccine strains in AIDSVAX B/E (MN.3.B and TH023.6.AE), a panel of heterologous Env-pseudotyped viruses that exhibit a tier 1 neutralization phenotype (see Supplemental Table 3 for additional details on the antigens used) or a tier 2 neutralization phenotype (Global Panel: 246-F3_C10_2, 25710-2.43, 398-F1_F6_20, BJOX002000.03.2, Ce1176_A3, Ce703010217_B6, CH119.10, CNE55, CNE8, TRO.11, X1632-S2-B10, and X2278_C2_B6). Response to a virus/isolate in the TZM-bl assay is considered positive if the neutralization titer is above a prespecified cutoff (one-half the lowest dilution tested). A titer was defined as the serum dilution that reduces relative luminescence units (RLUs) by $50 \%$ relative to the RLUs in virus control wells (cells + virus only) after subtraction of background RLU (cells only). The prespecified cutoff was 10 for TZM-bl cells.

Intracellular cytokine staining assay. Flow cytometry was used to examine HIV-1-specific $\mathrm{CD} 4^{+}$and $\mathrm{CD} 8^{+} \mathrm{T}$ cell responses using a val- idated intracellular cytokine staining (ICS) assay 2 weeks after each boost as well as 12 months after enrollment. The peptide pools evaluated were vaccine matched (ZM96 gp140-Env1, ZM96 gp140-Env2, 92TH023-Env, and ZM96 Gag), covering Env and Gag (Supplemental Table 3). Previously cryopreserved PBMCs were stimulated with the synthetic peptide pools. As a negative control, cells were not stimulated. As a positive control, cells were stimulated with a polyclonal stimulant, phytohemagglutinin (PHA). There were no replicates except for the negative control, which has 2 replicates.

Responses of $\mathrm{CD}^{+}$and $\mathrm{CD}^{+} \mathrm{T}$ cells measured by ICS for IL-2 and/or IFN- $\gamma$ to any HIV peptide pool (ZM96 gp140-Env 1, ZM96 gp140-Env 2, 92TH023-Env, and ZM96 Gag) are primary immunogenicity endpoints. ZM96 gp140-Env1 and -Env2 represent the N-terminal and C-terminal half of ZM96 gp140, respectively; since they were not overlapping, the response to ZM96 gp140 is represented as the sum of both pools. 92TH023-Env represents the full 92TH023 gp140; responses to "Any Env" are therefore represented as the maximum of ZM96 gp140-Env1 and -Env2 and 92TH023 Env. The response to "Any HIV protein" was defined as the sum of the response to Any Env and the response to Gag.

Several criteria were used to determine if data from an assay are acceptable and can be statistically analyzed. The blood draw date had to be within the allowable visit window as determined by the protocol. On the second day after sample thawing, the viability had to be $66 \%$ or greater. If not, the sample for that specimen at that time point had to be retested. If upon retesting the viability remained below this threshold, the ICS assay was not performed and no data were reported to the statistical center for that time point. For the negative control acceptance criteria, if the average cytokine response for the negative control wells was above $0.1 \%$ for either the $\mathrm{CD}^{+}{ }^{+}$or $\mathrm{CD} 8^{+} \mathrm{T}$ cells, then the sample had to be retested; it was the response for the retested sample that was analyzed.

The total number of $\mathrm{CD} 4^{+}$and $\mathrm{CD} 8^{+} \mathrm{T}$ cells had to exceed certain thresholds. If the number of $\mathrm{CD} 4^{+}$or $\mathrm{CD} 8^{+} \mathrm{T}$ cells was less than 5,000 for any of the HIV-1 peptide pools or one of the negative control replicates for a particular sample, data for that stimulation were filtered. If both negative control replicates contained fewer than 5,000 cells, the sample was retested. If upon retesting one negative control replicate contained fewer than 5,000 cells, the negative control replicate with more than 5,000 cells was used. If both negative control replicates from the retest for a $\mathrm{T}$ cell subset had fewer than 5,000 cells, then the data for the $\mathrm{T}$ cell subset were not included in the analysis.

$T$ cell $P F$ analyses. COMPASS is a computational framework for unbiased PF analysis of antigen-specific T cell subsets (41). COMPASS uses a Bayesian hierarchical framework to model all observed functional cell subsets and select those most likely to exhibit antigenspecific responses. Cell-subset responses were quantified by posterior probabilities, while participant-level responses were quantified by 2 summary statistics (functionality scores) that can be correlated directly with outcomes of interest, and describe the quality of an individual's functional response. The functionality score is defined as the estimated proportion of antigen-specific subsets detected among all possible ones. The PF score is similar, but it weighs the different subsets by their degree of functionality, naturally favoring subsets with higher degrees of functions, motivated by the observation that higher degree function has been correlated with good outcomes in certain vaccine studies. For this analysis, expression of IFN- $\gamma$, IL-2, TNF- $\alpha$, 
IL-4, and CD4OL was included. Scores were compared between treatment groups using Wilcoxon's rank-sum test. For Env-specific PF scores, overall responses across pools were computed by summing the cell counts, both for the number of expressing cells and the total cell count (numerator and denominator), in fitting a COMPASS model. For Any Env, these were summed for all 3 Env peptide pools. A heatmap for each stimulation and $\mathrm{T}$ cell subset shows the mean posterior probabilities of antigen-specific responses from COMPASS. Columns correspond to the different subsets of cytokines being considered and rows correspond to the mean across the individual participants in each treatment group at each time point. Each cell shows the probability that the corresponding antigen-specific subset (column) is being expressed in the corresponding treatment group in average (row), and is color coded ranging from white (zero) to dark purple (one).

VISR. VISR was assessed 2 weeks after the third and fourth vaccinations and at 9 and 12 months after enrollment using a diagnostic algorithm that includes 4 different enzyme immunoassays (EIAs): Abbott Architect HIV Ag/Ab Combo, Abbot Prism, Bio-Rad Genetic Systems HIV Combo Ag/Ab EIA, and Bio-Rad Multispot HIV-1/HIV-2 Rapid Test. For participants with a positive result in any of these assays, RNA PCR (Abbott m2000 HIV-1 Real-Time PCR) was performed to distinguish vaccine-induced responses from actual infection.

Statistics. The sample size of 26 vaccine recipients per group provided a $90 \%$ chance of observing at least $1 \mathrm{SAE}$ if the true rate of such an event was $8.5 \%$ or higher; there was a $90 \%$ chance that we would not observe any SAE if the true rate was less than $0.4 \%$. For immune responses, the precision with which the true response rate can be estimated from the observed data depended on the true underlying response rate and the sample size. For example, the 2-sided 95\% CI for the true response was approximately $67 \%-94 \%$ if the observed response rate was $85 \%$ based on the sample size of 26 vaccine recipients per group. The study had a limited power to detect response-rate differences between vaccine groups. The study could only detect a $30 \%$ or higher difference between vaccine groups with $80 \%$ power.

The percentages of participants experiencing each type of local and systemic reactogenicity sign or symptom are displayed using bar charts by severity and treatment group. For a given sign or symptom, each participant's reactogenicity events were counted once under the maximum severity. Kruskal-Wallis tests were used to test for differences in severity across all treatment groups.

The distributions of the immune-response magnitudes are displayed by treatment group and visit, with responders in red dots and nonresponders in blue triangles and box-and-whisker plots based on the data from responders superimposed on the distribution. The midline of the box-and-whisker plot denotes the median and the ends of the boxand-whisker plot denote the 25th and 75th percentiles. The whiskers that extended from the top and bottom of the box extend to the most extreme data points that were no more than 1.5 times the interquartile range (i.e., height of the box) or if no value meets this criterion, to the data extremes. The positive immune response rates are displayed in bar charts by treatment group and time point in separate panels.

Positive immune response rates were compared between treatment groups using Fisher's exact test and between visits within treatment groups using McNemar's test. The immune-response magnitudes among positive responders were compared between treatment groups using Wilcoxon's rank-sum exact test and between time points within treatment groups using Wilcoxon's signed-rank test.
The trajectory of $\operatorname{IgG} / \operatorname{IgA}$ and $\operatorname{IgG}$ subclass response rates over time are displayed by treatment group. The error bars at each time point are the $95 \% \mathrm{CI}$ of the response rate. The $95 \% \mathrm{CI}$ of the response rate was calculated using the score test method (42). The trajectory of IgG/ IgA and IgG subclass response magnitudes over time are displayed by treatment groups in geometric means among all participants (responders and nonresponders) from each treatment group. The error bars at each time point are the $95 \%$ CIs of the geometric means by treatment group. The $95 \% \mathrm{CI}$ of the geometric mean was calculated based on the normal distribution of $\log (\operatorname{IgG} / \operatorname{Ig} \mathrm{A})$ and back-transformation.

MB plots characterize the magnitude (nAb titer) and breadth (number of isolates neutralized at a given titer) of each individual serum sample assayed against a panel of isolates. MB curves show, for each possible $\log _{10}\left(\mathrm{ID}_{50}\right)$ titer threshold, the fraction of viruses with titers greater than this threshold. In addition to the individual samplespecific curves, the treatment-group-specific curve displays the average MB across all participants in that treatment group. The AUC-MB was calculated as the average of the $\log _{10}(\mathrm{nAb})$ titer over the panel of isolates, where titers that are below the limit of detection were set to half of that limit. The AUC-MB was compared between treatment groups using Wilcoxon's rank-sum exact test.

All $P$ values are 2 sided and unadjusted for multiple comparisons. Given the nature and sample size of this phase II trial $(n=26$ per treatment group), a somewhat increased type I (false positive) error without multiplicity correction was tolerated for better sensitivity (power) to detect the differences between treatment groups. Significant findings would generate the hypotheses for further testing in a larger trial in the future.

Study approval. The study was approved by the local institutional review boards (IRBs) at all participating sites: Human Research Protections Program/IRB, Vanderbilt University Medical Center Nashville, Tennessee, USA; University of Rochester Research Subject Review Board, Rochester, New York, USA; Fred Hutchinson Cancer Research Center IRB, Seattle, Washington, USA; UCSF Human Research Protection Program, San Francisco, California, USA; New York Blood Center, New York Blood Center IRB, New York, New York, USA; College of Physicians \& Surgeons, Columbia University IRB, New York, New York, USA; University of Pennsylvania IRB, Philadelphia, Pennsylvania, USA. Clinical research site staff completed enrollment procedures and obtained written informed consent from all participants.

Data availability. A copy of the study protocol and the data underlying the findings of this manuscript can be found online at https://atlas.scharp.org/cpas/project/HVTN\%20Public\%20Data/ HVTN\%20105/begin.view?

\section{Author contributions}

MCK, CM, NGR, RJ, SK, and ES designed the study. MES, IF, GJW, HVT, JM, AN, and MCK were responsible for the conduct of the trial. GDT, DCM, GF, SCDR, and MJM generated immunology data. SSL and BS conducted data analysis. FS, GP, and SD provided the vaccines used in the trial. NGR, MCK, and JK wrote the manuscript.

\section{Acknowledgments}

We thank all study participants enrolled in HVTN 105. We gratefully acknowledge the participation and support of many colleagues and staff on the HVTN 105 Protocol Team (see Supplemental Acknowledgments for consortium detail). At the clinical research 
sites we thank Beryl Koblin, Debbie Lucy, Leah Strock, Kiwan Stewart, Geneva Ortiz, Jermaine McCrossin, DaShawn Usher, David Berger, Kim Louis, Meredith Potochnic, Steven Palmer, Verna Robertson, Jorge Benitez, Marianella Rodriguez, Brett Gray, Ramon Negron, Hannah Catan, Lucy Liu, Deawodi Ladzekpo, Elizabeth Faber, Alfonso Diaz, Emily Schaeffer, Kimberly Marsh, Megan Henry, Nnenna Ozobia, Scott Fields, Monique Biega, Theresa Wagner, Debora Dunbar, Jie Ho, Annet David, Katie Crumbo, Cindy Hager, Robbie Maris, Naomi Prashad, Gwendolyn Rees, Rita Smith, Shonda Sumner, Kyle Rybczyk, and Spyros Kalams. For their roles in protocol development and study implementation, we thank Catherine Bunce, Laissa Ouedraogo, Hongwei Wang, Eva Chung, Gina Escamilla, Carissa Karg, Lisa Sunner, Renee Rivers, Angela Broad, Sheila Bello-Irizarry, Jim Maynard, Robert Bucklew, Adi Ferrara, Jill Zeller, Scharla Estep, and On Ho. For the binding-antibody assays and project and data management, we thank Kristy Long, Vicki Ashley, Judith Lucas, Mike Archibald, Yong Lin, R. Glenn Overman, Sheetal Sawant, Jack Heptinstall, Kelly Seaton, and David Beaumont. For the ADCC assays, we thank Sherry Stanfield-Oakley, Melissa Zinter, Charles Beck, and Katelyn Faircloth. For the neutralizing-antibody assays, we thank Amanda Eaton and Nathan Newton. For quality assurance oversight, we thank Marcella Sarzotti-Kelsoe. For the ICS assays, we thank Kristen Cohen and Nicole Frahm, and we thank Anthony Williams for performing the COMPASS analysis. We thank Barton Haynes, Hua-Xin Liao, James Peacock, and the Duke Protein Production Facility for protein reagents. For assistance with data management, programming, and analyses, we thank April Randhawa, Shannon Grant, Chenchen Yu, Mengshu Shao, Bhara- ti Lakshiminarayanan, Kyle Marshall, Cindy Molitor, and Sara Thiebaud. At GSID, we thank Carter Lee and Vineeta Gulati. At USMHRP, we thank Charla Andrews. For assistance with manuscript preparation, we thank Ashley Clayton and Mindy Miner. This work was supported by the National Institute of Allergy and Infectious Diseases (NIAID) US Public Health Service grants UM1 AI068614 (LOC: HIV Vaccine Trials Network), UM1 AI068635 (SDMC: HIV Vaccine Trials Network), UM1 AI068618 (LC: HIV Vaccine Trials Network), UM1 AI069511 (University of Rochester HIV/AIDS Clinical Trials Unit), UM1 AI069470 (Columbia Partnership for Prevention and Control of HIV/AIDS Clinical Trials Unit: College of Physicians \& Surgeons and New York Blood CenterClinical ResearchSites),UM1AI069534 and P30 AI450008 (Philadelphia HIV Therapeutics and Prevention Clinical Trials Unit), UM1 AI069439 (Vanderbilt), UM1 AI069481 (SeattleLausanne-Kampala Clinical Trials Unit), and UM1 AI069496 (San Francisco Bay Clinical Trials Unit). Additional support was provided by the National Center for Advancing Translational Sciences, NIH, through grant number UL1TR001873 (Columbia). The DNA vaccine was provided by the IPPOX Foundation, Switzerland, through support from the Collaboration of AIDS Vaccine Discovery of the Bill \& Melinda Gates Foundation grant OPP52845.

Address correspondence to: Nadine Rouphael, The Hope Clinic of the Emory Vaccine Center, Infectious Diseases Division, Department of Medicine, Emory University, 500 Irvin Court, Suite 200, Decatur, Georgia 30030, USA. Phone: 404.712.1435; Email:nroupha@emory.edu.
1. [No authors listed]. HIV/AIDS fact sheet. World Health Organization. http://www.who.int/ mediacentre/factsheets/fs360/en/. Updated July 19, 2018. Accessed August 21, 2019.

2. Rerks-Ngarm S, et al. Vaccination with ALVAC and AIDSVAX to prevent HIV-1 infection in Thailand. N Engl J Med. 2009;361(23):2209-2220.

3. Pitisuttithum P, et al. Randomized, double-blind, placebo-controlled efficacy trial of a bivalent recombinant glycoprotein 120 HIV-1 vaccine among injection drug users in Bangkok, Thailand. JInfect Dis. 2006;194(12):1661-1671.

4. Flynn NM, et al. Placebo-controlled phase 3 trial of a recombinant glycoprotein 120 vaccine to prevent HIV-1 infection. J Infect Dis. 2005;191(5):654-665.

5. Haynes BF, et al. Immune-correlates analysis of an HIV-1 vaccine efficacy trial. $N$ Engl JMed. 2012;366(14):1275-1286.

6 . Jin X, et al. Multiple factors affect immunogenicity of DNA plasmid HIV vaccines in human clinical trials. Vaccine. 2015;33(20):2347-2353.

7. Yates NL, et al. Vaccine-induced Env V1-V2 IgG3 correlates with lower HIV-1 infection risk and declines soon after vaccination. Sci Transl Med. 2014;6(228):228ra39.

8. Tomaras GD, et al. Vaccine-induced plasma IgA specific for the $\mathrm{C} 1$ region of the HIV-1 envelope blocks binding and effector function of IgG. Proc Natl Acad Sci U S A. 2013;110(22):9019-9024.

9. Goepfert PA, et al. Specificity and 6-month dura- bility of immune responses induced by DNA and recombinant modified vaccinia Ankara vaccines expressing HIV-1 virus-like particles. J Infect Dis. 2014;210(1):99-110.

10. Hammer SM, et al. Efficacy trial of a DNA/ rAd5 HIV-1 preventive vaccine. $N$ Engl J Med. 2013;369(22):2083-2092.

11. Spearman P, et al. A trimeric, V2-deleted HIV-1 envelope glycoprotein vaccine elicits potent neutralizing antibodies but limited breadth of neutralization in human volunteers. J Infect Dis. 2011;203(8):1165-1173.

12. Wang $\mathrm{S}$, et al. Cross-subtype antibody and cellular immune responses induced by a polyvalent DNA prime-protein boost HIV-1 vaccine in healthy human volunteers. Vaccine. 2008;26(8):1098-1110.

13. Koopman G, et al. Immune-response profiles induced by human immunodeficiency virus type 1 vaccine DNA, protein or mixed-modality immunization: increased protection from pathogenic simian-human immunodeficiency virus viraemia with protein/DNA combination. JGen Virol. 2008;89(Pt 2):540-553.

14. Patel V, et al. DNA and virus particle vaccination protects against acquisition and confers control of viremia upon heterologous simian immunodeficiency virus challenge. Proc Natl Acad Sci U S A. 2013;110(8):2975-2980.

15. Hessell AJ, et al. Achieving potent autologous neutralizing antibody responses against tier 2 HIV-1 viruses by strategic selection of envelope immunogens. JImmunol. 2016;196(7):3064-3078.

16. Jalah R, et al. DNA and protein co-immunization improves the magnitude and longevity of humoral immune responses in macaques. PLOS ONE. 2014;9(3):e91550.

17. Li J, et al. HIV/SIV DNA vaccine combined with protein in a co-immunization protocol elicits highest humoral responses to envelope in mice and macaques. Vaccine. 2013;31(36):3747-3755.

18. Gao F, et al. Antigenicity and immunogenicity of a synthetic human immunodeficiency virus type 1 group m consensus envelope glycoprotein. JVirol. 2005;79(2):1154-1163.

19. Liao HX, et al. A group M consensus envelope glycoprotein induces antibodies that neutralize subsets of subtype B and C HIV-1 primary viruses. Virology. 2006;353(2):268-282.

20. Corey L, Gilbert PB, Tomaras GD, Haynes BF, Pantaleo G, Fauci AS. Immune correlates of vaccine protection against HIV-1 acquisition. Sci Transl Med. 2015;7(310):310rv7.

21. Chung AW, et al. Polyfunctional Fc-effector profiles mediated by IgG subclass selection distinguish RV144 and VAX003 vaccines. Sci Transl Med. 2014;6(228):228ra38.

22. Tay MZ, et al. Antibody-mediated internalization of infectious HIV-1 virions differs among antibody isotypes and subclasses. PLoS Pathog. 2016;12(8):e1005817.

23. Chung AW, et al. Dissecting polyclonal vaccine- 
induced humoral immunity against HIV using systems serology. Cell. 2015;163(4):988-998.

24. Banerjee K, et al. IgG subclass profiles in infected HIV type 1 controllers and chronic progressors and in uninfected recipients of Env vaccines. AIDS Res Hum Retroviruses. 2010;26(4):445-458.

25. Gorse GJ, et al. HIV-1MN recombinant glycoprotein 160 vaccine-induced cellular and humoral immunity boosted by HIV-1MN recombinant glycoprotein 120 vaccine. National Institute of Allergy and Infectious Diseases AIDS Vaccine Evaluation Group. AIDS Res Hum Retroviruses. 1999;15(2):115-132.

26. Rerks-Ngarm S, et al. Randomized, doubleblind evaluation of late boost strategies for HIV-uninfected vaccine recipients in the RV144 HIV vaccine efficacy trial. J Infect Dis. 2017;215(8):1255-1263.

27. Hendrikx LH, et al. Different IgG-subclass distributions after whole-cell and acellular pertussis infant primary vaccinations in healthy and pertussis infected children. Vaccine. 2011;29(40):6874-6880.

28. Vasan S, et al. In vivo electroporation enhances the immunogenicity of an HIV-1 DNA vaccine candidate in healthy volunteers. PLoS One. 2011;6(5):e19252.

29. Nilsson C, et al. HIV-DNA given with or without intradermal electroporation is safe and highly immunogenic in healthy Swedish HIV-1 DNA/ MVA vaccinees: A phase I randomized trial. PLOS ONE. 2015;10(6):e0131748.

30. Li SS, et al. DNA priming increases frequency of T-cell responses to a vesicular stomatitis virus HIV vaccine with specific enhancement of $\mathrm{CD}^{+} \mathrm{T}$-cell responses by interleukin-12 plasmid DNA. Clin Vaccine Immunol. 2017;24(11):e00263-17.

31. Lee J, Arun Kumar S, Jhan YY, Bishop CJ. Engineering DNA vaccines against infectious diseases. Acta Biomater. 2018;80:31-47.

32. Gaudinski MR, et al. Safety, tolerability, and immunogenicity of two Zika virus DNA vaccine candidates in healthy adults: randomised, open-label, phase 1 clinical trials. Lancet. 2018;391(10120):552-562.

33. Singh M, et al. A preliminary evaluation of alternative adjuvants to alum using a range of established and new generation vaccine antigens. Vaccine. 2006;24(10):1680-1686.

34. Jackson LA, et al. Randomized clinical trial of a single versus a double dose of 13 -valent pneumococcal conjugate vaccine in adults 55 through 74 years of age previously vaccinated with 23-valent pneumococcal polysaccharide vaccine. Vaccine. 2018;36(5):606-614.

35. Osterholm MT, Kelley NS, Sommer A, Belongia EA. Efficacy and effectiveness of influenza vac- cines: a systematic review and meta-analysis. Lancet Infect Dis. 2012;12(1):36-44.

36. Bull M, et al. Defining blood processing parameters for optimal detection of cryopreserved antigen-specific responses for HIV vaccine trials. J Immunol Methods. 2007;322(1-2):57-69.

37. Tomaras GD, et al. Initial B-cell responses to transmitted human immunodeficiency virus type 1: virion-binding immunoglobulin $\mathrm{M}(\operatorname{IgM})$ and IgG antibodies followed by plasma anti-gp41 antibodies with ineffective control of initial viremia. J Virol. 2008;82(24):12449-12463.

38. Alam SM, et al. Antigenicity and immunogenicity of RV144 vaccine AIDSVAX clade E envelope immunogen is enhanced by a gp120 N-terminal deletion. J Virol. 2013;87(3):1554-1568.

39. Pollara J, et al. High-throughput quantitative analysis of HIV-1 and SIV-specific ADCCmediating antibody responses. Cytometry $A$. 2011;79(8):603-612.

40. Montefiori DC. Measuring HIV neutralization in a luciferase reporter gene assay. Methods Mol Biol. 2009;485:395-405.

41. Lin L, et al. COMPASS identifies T-cell subsets correlated with clinical outcomes. Nat Biotechnol. 2015;33(6):610-616.

42. Agresti A, Coull BA. Approximate is better than "exact" for interval estimation of binomial proportions. Am. Sta. 1998;52(2):119-126. 